\title{
Deterministic Finite Automata Characterization and Optimization for Scalable Pattern Matching
}

\author{
LUCAS VESPA and NING WENG, Southern Illinois University Carbondale
}

\begin{abstract}
Memory-based Deterministic Finite Automata (DFA) are ideal for pattern matching in network intrusion detection systems due to their deterministic performance and ease of update of new patterns, however severe DFA memory requirements make it impractical to implement thousands of patterns. This article aims to understand the basic relationship between DFA characteristics and memory requirements, and to design a practical memory-based pattern matching engine. We present a methodology that consists of theoretical DFA characterization, encoding optimization, and implementation architecture. Results show the validity of the characterization metrics, effectiveness of the encoding techniques, and efficiency of the memory-based pattern engines.
\end{abstract}

Categories and Subject Descriptors: C.3 [Special-Purpose and Application-based Systems]; I.5.5 [Pattern Recognition]: Implementation-Special architectures

General Terms: Design, Security

Additional Key Words and Phrases: Pattern matching, deterministic finite automata, encoding optimization ACM Reference Format:

Vespa, L. and Weng, N. 2011. Deterministic finite automata characterization and optimization for scalable pattern matching. ACM Trans. Architec. Code Optim. 8, 1, Article 4 (April 2011), 31 pages.

DOI $=10.1145 / 1952998.1953002 \mathrm{http} / / /$ doi.acm.org/10.1145/1952998.1953002

\section{INTRODUCTION}

Pattern matching is playing an increasingly important role in bioengineering for DNA sequence matching [Herisson et al. 2007; Soewito and Weng 2007; Lu et al. 2006] and network applications for payload scanning (virus scanner, spam filters, and network intrusion detection [Tuck et al. 2004; Denning 1987; Roesch 1999; Varghese 2005; $\mathrm{Bu}$ and Chandy 2004]). For example, network intrusion detection systems detect and thwart a network attack by comparing each incoming packet with predefined patterns, called an attack pattern database, and raise an alert upon detecting a match. Although a substantial amount of work has been proposed in the area of pattern matching for network intrusion detection, most of the existing work does not meet the challenges of the next generation of pattern matching: performance scalability and easy pattern update [Varghese 2005; Tan et al. 2006].

Deterministic Finite Automata (DFA) [Aho and Corasick 1975; Brodie et al. 2006; Soewito et al. 2009; Vespa et al. 2009] is able to match multiple strings simultaneously, in worst-case time linear to the size of a packet. Unfortunately, DFA are difficult to implement effectively as they possess many characteristics that make them spatially complex and memory inefficient [Becchi and Crowley 2007; Kumar et al. 2006; van Lunteren 2006; Smith et al. 2008b; Vespa and Weng 2009b]. Many attempts have been

Authors' address: L. Vespa and N. Weng, Department of Electrical and Computer Engineering, 1230 Lincoln Drive, Southern Illinois University Carbondale, Carbondale, IL 62901; email: \{vespa, nweng\}@siu.edu. Permission to make digital or hard copies of part or all of this work for personal or classroom use is granted without fee provided that copies are not made or distributed for profit or commercial advantage and that copies show this notice on the first page or initial screen of a display along with the full citation. Copyrights for components of this work owned by others than ACM must be honored. Abstracting with credit is permitted. To copy otherwise, to republish, to post on servers, to redistribute to lists, or to use any component of this work in other works requires prior specific permission and/or a fee. Permissions may be requested from Publications Dept., ACM, Inc., 2 Penn Plaza, Suite 701, New York, NY 10121-0701 USA, fax +1 (212) 869-0481, or permissions@acm.org.

(C) 2011 ACM 1544-3566/2011/04-ART4 $\$ 10.00$

DOI 10.1145/1952998.1953002 http://doi.acm.org/10.1145/1952998.1953002 


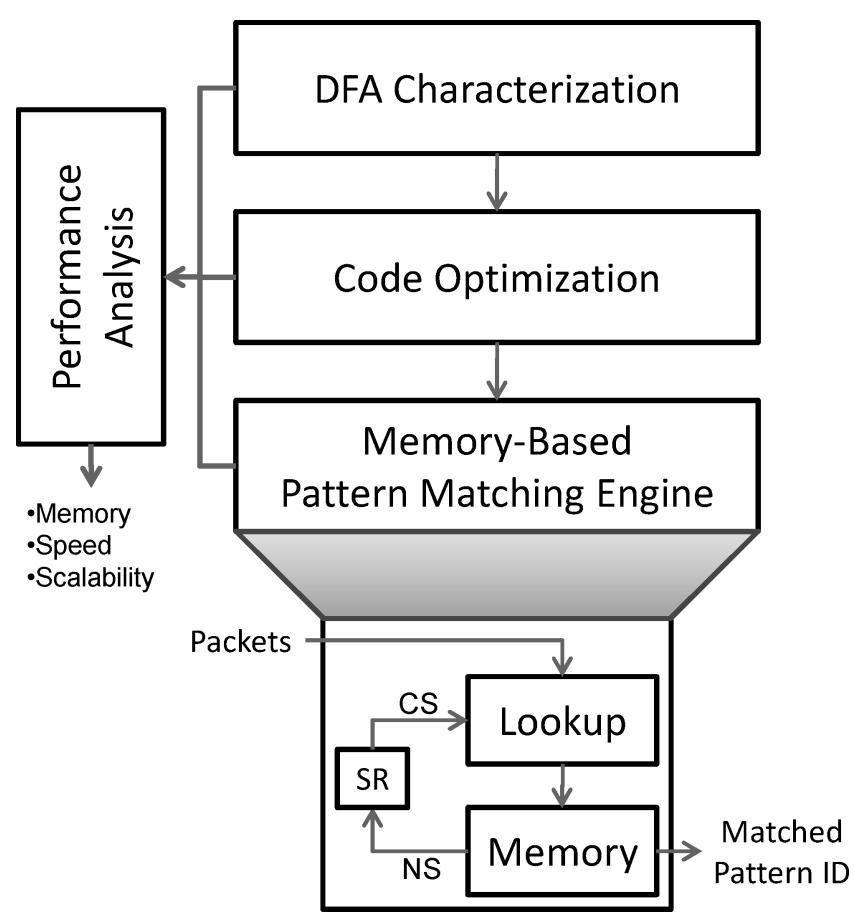

Fig. 1. Memory-based pattern matching engine design methodology.

made to remedy DFA space issues but most are based on observations made about DFA test-sets and trends. Therefore, some proposed solutions either present negative performance tradeoffs, or limit the scope of patterns that DFA can be constructed from. However, there are certain DFA characteristics that when exploited properly, can supersede these limitations.

This article presents a theoretical analysis of the characteristics of DFA that cause complexity and memory issues, coding techniques for optimizing memory-based DFA for memory and performance, as well as design cases of memory-based engines. Figure 1 illustrates these components and their relationships. DFA characterization is used to understand DFA properties that are in turn used to develop encoding optimization techniques. These encoding techniques optimize the memory content of the final memory-based pattern matching engines. Performance analysis is used to evaluate our techniques and engines. The three main components are further described as follows.

(1) DFA characterization is a theoretic analysis of the relationship of DFA properties with memory and performance. This analysis is necessary for developing optimizations that benefit from the full spectrum of DFA characteristics. It is difficult to fully characterize DFA in that many properties of DFA are indirectly related to other properties in either an orthogonal or parallel manner. Our theoretic analysis differentiates itself from previous work by using several simple metrics that encapsulate the relationship between states, symbols, and transitions. We present three DFA metrics: state independence, character distribution, and state irregularity.

(2) Encoding optimization aims at optimizing the state codes stored in memory-based DFA, rather than just optimizing the DFA itself. This is different than previous approaches, which concentrate on reducing DFA transitions. Our encoding techniques are designed to optimize the previously discussed characteristics. We present three 
optimization techniques: self-addressable state encoding, character-aware encoding, and split-level.

(3) Memory-based engine is the actual implementation of DFA in memory, of which we design three implementations that exploit the concepts and optimizations discussed in this article. The memory-based engines utilize a finite state machine (FSM) based structure, as seen in Figure 1. It consists of a memory array that stores the states for DFA, a state register (SR) that stores the current state (CS), and instructions for lookup that contain the logic to find the next state (NS) in memory using the current state and the input (a character or symbol). Specifically, three novel memory-based engines are proposed: Self Addressable Memory-Based Finite State Machine (SAMFSM), Portable Predictive Pattern Matching Finite State Machine $\left(P^{3} F S M\right)$, and Simple Instruction Finite State Machine (SI-FSM).

With the methodology in Figure 1, we are able to better understand the relationship between DFA characteristics and memory, efficiently optimize DFA to improve those characteristics and design an efficient pattern matching engine. In summary, the three main contributions of this work are the following.

(1) DFA Characterization Metrics

- State Independence. estimates the coding length due to state dependence;

-Character Distribution quantifies the time complexity of lookup operations due to label distribution;

-State Irregularity. measures memory waste due to irregular edge distribution.

(2) Encoding Optimization Techniques

-Self-Addressable Encoding. achieves a high level of memory compression by analyzing dependency between states

-Character Aware Coding. allows next state lookup to be performed in a single comparison without requiring address decoding;

—Split-Level. DFA optimization for maximizing state independence and minimizing character distribution for memory compression and lookup time (single or multi split).

(3) Pattern Matching Engine Architectures

- SAM-FSM. uses self-addressable encoding;

$-P^{3}$ FSM. uses character-aware encoding and split-level (single);

-SI-FSM. uses character-aware encoding and split-level (multiple).

This DFA characterization methodology does not specifically target strings or regular expressions, but rather existing DFA. We do not directly address construction of regexbased DFA, particularly the state explosion problem [Smith et al. 2008b]. However, Section 2.4 discusses the differences between DFA built from exact strings and DFA built from regular expressions. We also show the benefits of our encoding methods on regular expression DFA in Sections 6.2 and 6.3.

Our results verify our DFA characterization by analyzing DFA built from Snort [2009] rulesets. We then analyze our encoding optimizations in terms of memory and performance and compare our techniques to other approaches. We evaluate the memory and performance benefits our memory-based pattern matching engines. Our results demonstrate the effectiveness of our methodology for designing a memory-based pattern matching engine. In particular, we present the following results.

- Our characteristic metrics are used to evaluate different DFA and their optimizations for memory and performance.

-Our encoding optimization techniques are examined to see how they affect the aforementioned DFA characteristics. 


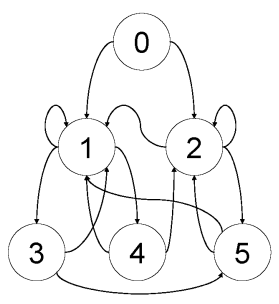

(a) Graph

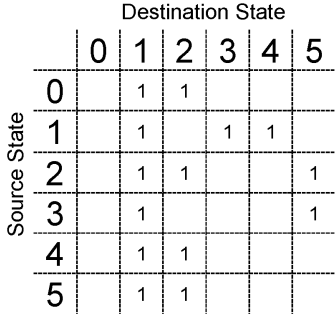

(b) Adjacency matrix

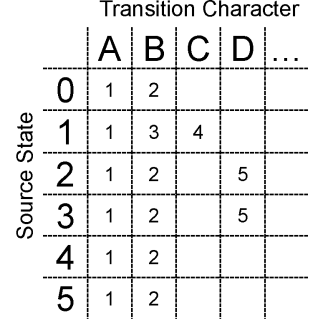

(c) Traditional matrix

Fig. 2. Deterministic finite automata (DFA) representations.

-Our memory-based engines are evaluated and compared against other memory-based DFA implementations.

The remainder of the article is organized as follows. The background of DFA and usage for pattern matching are introduced in Section 2. Sections 3, 4, and 5 present DFA characterization metrics, encoding techniques, and implementation engines respectively. Results are shown and discussed in Section 6. We discuss related work in Section 7. Section 8 concludes the article.

\section{DETERMINISTIC FINITE AUTOMATA}

Deterministic finite automata (DFA) are used extensively for memory-based pattern matching due to their deterministic processing. This section presents background information about DFA as well as challenges in implementing memory-based DFA. Section 2.1 presents a formal description of DFA and Section 2.2 shows examples of how to represent a DFA with a graph and a matrix, both of which are used in this article. Section 2.3 presents an analysis of memory-based DFA and the challenges with DFA memory inefficiency, as well as an overview of the complexity of DFA operations that affect memory-based DFA performance. Section 2.4 discusses the structural differences between string and regular-expression DFA.

\subsection{DFA Description}

A Deterministic Finite Automaton (D) is defined as $\mathrm{D}=\left\{\mathrm{Q}, \mathrm{C}, \delta, q_{0}, \mathrm{~F}\right\}$, where:

$-\mathrm{Q}$ is a finite set of states.

$-\mathrm{C}$ is a finite set of input symbols.

- $\delta$ is a transition function from $Q \times C \rightarrow Q$ for all $q_{i} \in Q$.

$-q_{0}$ is the initial state and $q_{0} \in Q$.

$-\mathrm{F}$ is the set of matching states and $F \subseteq Q$.

The operation of $\delta$ is deterministic and therefore, a maximum of one $q_{j}$ exists for any $\delta\left(q_{i}, c\right) \rightarrow q_{j}$. This means there is only one outgoing transition per character per state, at the most. Also, $\delta\left(q_{i}, c\right) \rightarrow\{\emptyset\}$ for any $c \notin C$. A result of $\{\emptyset\}$ resets to the initial state, $q_{0}$.

\subsection{DFA Representation}

A DFA can be represented using multiple methods depending on the purpose of the representation. In order to help convey ideas about DFA, we may represent them as a visual graph, as seen in Figure 2(a). This figure shows a DFA with $|Q|=6$. Each directed edge represents $\delta\left(q_{\text {source }}, c_{\text {dest }}\right) \rightarrow q_{\text {dest }}$, or a transition from a source state to a destination state on an input character of the destination state. The characters or 
symbols in $C$ are not shown in this example graph, however, a transition to any state is a conditional transition assuming a specific input character.

We can also use an adjacency matrix as representation, which is helpful in mathematically extracting properties of DFA. Figure 2(b) shows an adjacency matrix that represents $\delta$ of Figure 2(a). Each row represents a source state and each column a destination state. If the intersection of any row and column contains a one, there exists at least one $c \in C$ such that $\delta\left(q_{\text {row }}, c\right) \rightarrow q_{c o l}$. If an intersection does not contain a one then $\delta\left(q_{\text {row }}, c_{\text {col }}\right) \rightarrow\{\emptyset\}$.

Another DFA matrix representation is a traditional matrix, as shown in Figure 2(c). This matrix represents all of the labeled transitions in a DFA. Each row corresponds to the current state and each column to a possible input character. At the intersection of a state and character, is the next state for that current state and input character. For example, in Figure 2(c), if the current state is one (e.g., row labeled 1), and the input character is $\mathrm{C}$ (e.g., column labeled C), then the next state is 4 . This matrix is very similar to how a DFA is traditionally stored in memory. The next state for each possible current state/input character combination is stored in memory. This produces prohibitive memory requirements.

\subsection{Memory-Based DFA}

The task of implementing DFA in memory consists of mapping the transition function $\delta$ into memory space. The transition function defines the next states $\delta\left(q_{i}, c_{j}\right)$ for current state $q_{i}$ and input character $c_{j}$. In a standard memory-based DFA, each current state $q_{i}$ has a code in memory and that code contains $\delta\left(q_{i}, c_{j}\right)$ for all 256 values of $j$. Therefore, each current state's code contains 256 next-state values. Each next-state in the code $q_{i}$ requires $\left\lceil\log _{2}|Q|\right\rceil$ bits so that each state has a unique value. There is one code in memory for each state $q \in|Q|$ states. This makes the total memory requirement for a memory-based DFA $\left(|Q| \cdot\left\lceil\log _{2}|Q|\right\rceil \cdot 256\right)$. This memory requirement is only practical when implementing a DFA from a few patterns, not for large pattern sets.

DFA optimizations must be employed to reduce this memory requirement for large pattern sets. When a DFA optimization is applied, transitions are removed from the DFA so that each state no longer has $|C|$ outgoing transitions. Obviously, the aforementioned structure cannot be used to implement an optimized DFA, as the benefits of the optimization would be nullified. The codes for each state for the optimization must be stripped down to only contain the next states in the optimized DFA. Figure 3 shows how the codes would be stored in this manner. Each code has entries $\left\{E_{1}, E_{2}, \ldots E_{k}\right\}$, where $k$ is the maximum fan-out for any state; therefore $k$ is the maximum number of entries in the codes. The goal here is to store only the transitions that remain after transition reductions. In order to do so we must store the appropriate character for each transition as shown by $(q, c)$ in Figure 3.

Each entry $E_{i}$, contains one next-state for any given current state. Because the code for each current state $q_{i}$ no longer contains 256 entries each, the lengths of the codes for different states are not the same. We denote the length of the code word for state $i$ as $L_{q_{i}}$ and the length of the longest code word as $L_{\max }$. For example, in Figure $3, L_{q_{0}}$ is less than $L_{q_{1}}$ because $q_{0}$ only has two next states whereas $q_{1}$ has many. The problem here is that $L_{\max }$ controls the memory width of all codes, which means that the total memory requirement is $\left(L_{\max } \cdot|Q|\right)$. Unused entries in the code are wasted memory. This problem is characterized by our metric called state irregularity in Section 3.3 and is solved by our split-level optimization in Section 4.3. Split-level makes each code more regular and therefore reduces memory waste by reducing $L_{\max }$.

Another method to shorten $L_{\max }$ is alphabet reduction [Becchi and Crowley 2007]. The number of characters and number of bits used to represent each character can be reduced, which in turn reduces the entries in each code, as well as reducing $L_{\max }$. 


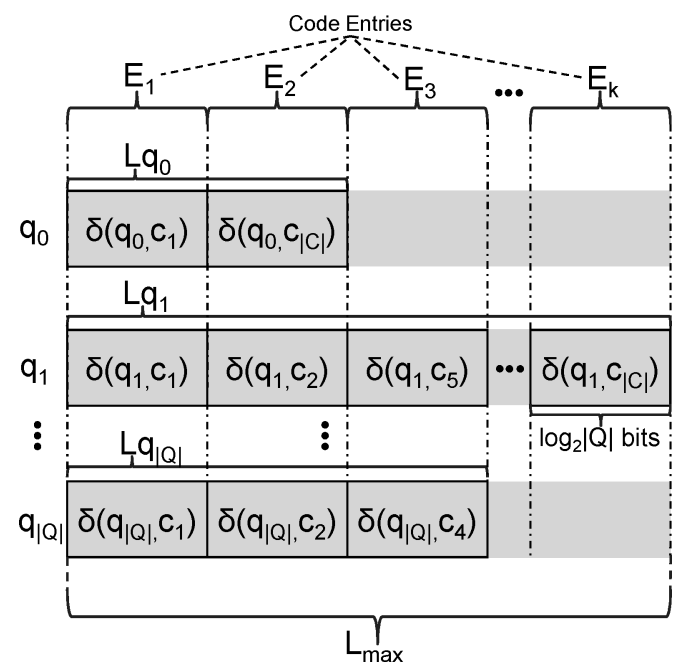

Fig. 3. In a memory-based DFA, each state $q_{i}$ has a code that contains all of its possible next states. Each code has up to $k$ next-states stored in entries $E_{1}$ through $E_{k}$ where $k$ is the maximum fan-out of the DFA. Each state has length $\mathrm{L} q_{i}$, and the longest code has length $L_{\max }$.

Our character-aware encoding is a form of alphabet reduction, wherein the number of characters and number of bits for each character is reduced. With character-aware encoding, it often only requires 4 bits to represent a set of about 100 characters, which normally requires 7 bits.

Another problem with memory-based DFA is that each entry $E_{i}$, in the state codes in Figure 3 requires $\left\lceil\log _{2}|Q|\right\rceil$ bits to represent a state. This causes $L_{\max }$ to be very long. This problem is characterized by our metric state independence in Section 3.1 and solved by self-addressable encoding in Section 4.1, such that only $\left\lceil\log _{2}\left|E_{i}\right|\right\rceil$ bits are required to represent entry $E_{i}$ in state codes.

In Figure 3, the current state $q_{i}$ indicates which row contains the next state. Given an input character $c_{j}$, in order to find the next state we must locate the column or entry corresponding to $c_{j}$ within row $q_{i}$. Up to $k$ entries of row $q_{i}$ must be searched in order to find the entry corresponding to $c_{j}$, and without the aid of special hardware, the entries must be searched sequentially one by one. This problem is characterized by our metric character distribution in Section 3.2 and is solved by the encoding optimization called character-aware coding in Section 4.2. Character-aware coding only allows all next-states for character $c_{j}$ to be stored in the same entry making next-state lookup $\mathrm{O}(1)$ rather than $\mathrm{O}(\mathrm{k})$.

\subsection{Regular-Expression DFA}

In our work, we analyze and optimize existing DFA, making our methodology applicable to any type of DFA. However, there are distinct differences between DFA constructed from exact strings and those constructed from regular expressions. These differences can cause different resulting values for our metrics as well as different memory requirements for our encoding schemes. These differences are caused by regular-expression operators, which allow for the creation of more complex search parameters. These operators result in a different DFA structure in terms of number of states and edge distribution.

2.4.1. Regular-Expression Operators. The main operators [Yu et al. 2006] used by regularexpressions are wildcards (e.g., '.'), repetition (e.g., '?', '+') and character classes (e.g., 
'l', '[]'). For example, the operator '*' denotes zero or more of the previous character. So the regular expression "ab*c" represents the patterns "ac," "abc," "abbc," and so on. A regular expression " $a(a \mid b) c$ " using the "or" operator represents the strings "aac" and "abc." Regular expressions with character classes can cause transitions with many different labels to converge to the same state, increasing DFA complexity. Another added level of complexity comes from counters, which are finite repetition operators.

2.4.2. Regular-Expression DFA. The complexity of regular-expression operators creates structural differences when compared to string-based DFA. The main structural differences that affect the results of our analysis and optimization are convergence [Yu et al. 2006], looping [Smith et al. 2008a] and state replication [Smith et al. 2008b].

Convergence describes when a state receives transitions on many different labels or characters. This happens when a regular expression uses a character class, or when multiple states converge. This affects the value of our character distribution metric and the memory requirement of our character-aware encoding technique.

Loops are created when a state transitions to itself or to a predecessor state. These loops commonly occur when using repetition operators. Loops affect the value of our state independence and state irregularity metrics.

State replication describes when a set of states is copied within a DFA. State replication especially occurs when using counters and wildcard operators. The amount of replication can be small or large. For example, if the '.' operator is used for a single character, this might cause a few states to be replicated. The end result of this is just a small increase in memory requirements due to an increase in states, and does not have a severe impact on any metrics or optimizations. However, if large value counters (e.g., several to hundreds of repeated strings) or a repeated wildcard (e.g., ‘.') are used, then the replication can be severe enough that memory requirements explode prohibitively. This large amount of replication cannot be addressed by transition reduction schemes and must be treated specially [Smith et al. 2008b]. Dealing with large state replication is an orthogonal problem to our methodology. We assume that a large amount of state replication is diverted by splitting the patterns in order to avoid replication. This optimization must be implemented before DFA construction, whereas our methodology focuses on post DFA construction.

\section{DFA CHARACTERIZATION}

This section presents a theoretical analysis of the characteristics of DFA that cause performance and memory issues using three metrics: State Independence, Character Distribution and State Irregularity. Each metric is an analysis tool for different DFA characteristics, and can also be used to evaluate the effectiveness of DFA optimizations and DFA encoding techniques.

State independence is necessary because relations between states caused by DFA transitions create a large memory requirement. State independence estimates the state coding length due to these state relations, allowing us to evaluate the effectiveness of the memory reduction ability of encoding techniques. Our approach for approximating state independence is to find the number of states that receive fan-in transitions from many states, which we call dependent states. These states are the biggest contributors to long state codes.

Character distribution is needed because the distribution of labeled edges in DFA can cause an extended next-state lookup time. Character distribution quantifies the time complexity of lookup operations due to label distribution. This allows us to evaluate how well an encoding technique increases the performance of DFA. We approximate character distribution by counting the occurrences and dependence between different 


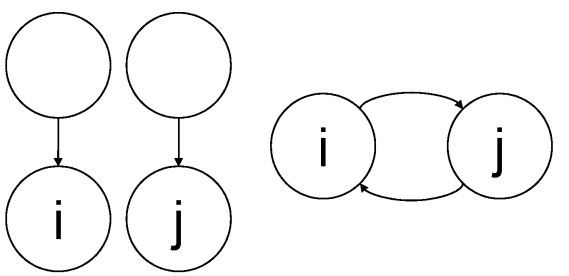

(a) Independent

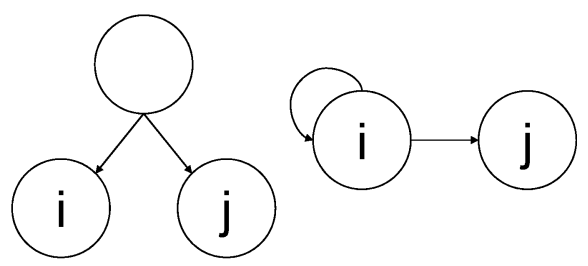

(b) Dependent

Fig. 4. Example of independent and dependent states. Two states are independent if they do not share any fan-in state.

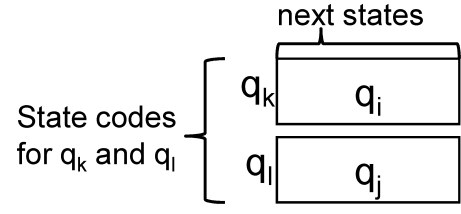

(a) Independent states $q_{i}$ and $q_{j}$ The state code for $q_{k}$ has next state $q_{i}$ and the state code for $q_{l}$ has next state $q_{j} . q_{i}$ and $q_{j}$ are independent so they can be stored in the same entry of $q_{k}$ and $q_{l}$.

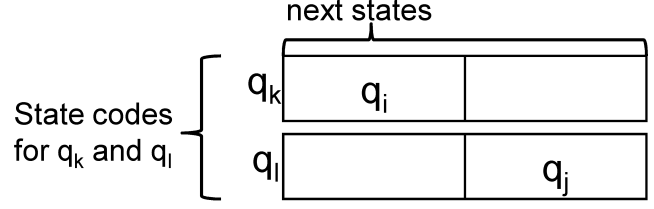

(b) Dependent states $q_{i}$ and $q_{j}$ The state code for $q_{k}$ has next state $q_{i}$ and the state code for $q_{l}$ has next state $q_{j} . q_{i}$ and $q_{j}$ are dependent and must be stored in different entries of $q_{k}$ and $q_{l}$. This extends the length of the state codes.

Fig. 5. Example of how state independence affects the length of state codes.

characters in DFA. This tells us approximately how many entries must be checked on average to find the next state for any given character.

State irregularity is necessary because DFA has a natural imbalance in the distribution of edges, often causing severe memory waste. State irregularity measures memory waste due to irregular edge distribution, and can be used to evaluate the effectiveness of DFA optimizations such as transition reduction schemes. State irregularity is approximated by finding the variance in the average fan-in for all states. This tells us approximately how much of each state code word is unused, or wasted, on average.

\subsection{State Independence}

Two states $q_{i}$ and $q_{j}$ are independent if they do not share any fan-in state. Figure 4(a) shows two states $i$ and $j$ that are independent, whereas Figure 4(b) shows states $i$ and $j$ that are dependent. If two states $q_{i}$ and $q_{j}$ share one fan-in state $q_{k}$, then states $q_{i}$ and $q_{j}$ cannot be in the same entry because they correspond to two different characters. However, if two states are independent (sharing no fan-in state), then both values can be stored in the same entry $E_{i}$. An example of how state independence affects state codes is shown in Figure 5.

We can estimate the excess percentage of $L_{\max }$ that is caused by states being dependent by using Equation (1). $S D$ gives a general metric for measuring state dependence (not independence) for a given DFA. As $S D$ approaches zero, a DFA contains very little dependence and will yield a low width for $L_{\max }$, whereas a value of close to one for $S D$, 


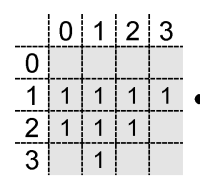

$[\mathrm{A}]^{\mathrm{T}}$

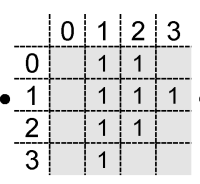

[A]

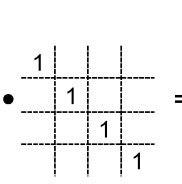

[I]

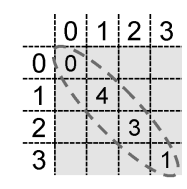

$\mathrm{FI}_{\text {deg }}$

Fig. 6. Calculating fan-in degree sequence using adjacency matrix.

indicates much more dependency and will yield a longer $L_{\max }$.

$$
S D=1-\frac{\left\lceil\log _{2}\left(|Q|-Q_{d e p}\right)\right\rceil}{Q_{d e p}+\left\lceil\log _{2}\left(|Q|-Q_{d e p}\right)\right\rceil} .
$$

In Equation (1), $Q_{d e p}$ is the number of states in a DFA that are nearly completely dependent. In order to estimate $Q_{d e p}$, we start with adjacency matrix [A] to represent transition function $\delta$. If we define the fan-in transitions for any state $q_{i}$ as all $\delta\left(q_{j}, c_{i}\right) \rightarrow$ $q_{i}$, where $j$ is all rows in column $i$ of [A] that contain ones, then the fan-in degree sequence $\left(F I_{d e g}\right)$ for $Q$ can be calculated by Equation (2). In Equation (2), $I$ is an identity matrix. Figure 6 shows an example of this calculation. The diagonal of the resulting matrix is the fan-in degree for each state $q_{r o w}$. This degree sequence can then be used in Equation (3) to estimate dependent states.

$$
\begin{aligned}
& F I_{d e g}=[A]^{T} \cdot[A] \cdot I . \\
& Q_{d e p}=\frac{\sum_{i=1}^{|Q|} F I_{d e g}(i)}{|Q|} .
\end{aligned}
$$

\subsection{Character Distribution}

Character distribution can be used to analyze the complexity of DFA lookup operations. For example, let us use the notation $E^{c}$ to represent the set of entries belonging to character c. Then $\left|E^{c}\right|$ is the number of sequential lookups that must be performed for input character $c .\left|E^{c}\right|$ is affected by the distribution of transitions with label $c$.

A metric for estimating the effect of character distribution $(C D)$ can be developed with the aid of the fan-in degree sequence derived in the previous section. In Equation (4), $\left(R_{c}\right)$ is the percentage of all states that have outgoing transitions on character c. In this Equation $Q^{c}$ is the set of states whose incoming transitions occur on character $c$. The character distribution metric $C D$ can then be calculated by Equation (5). As $C D$ approaches zero, the effect of character distribution on lookup is small. As $C D$ approaches one, the effect is large, which means that next state lookup will be a large sequence of sequential lookups, which is very time consuming. This can be remedied by an encoding optimization called character-aware, which is discussed in the encoding optimizations section.

$$
\begin{gathered}
R_{c}=\sum_{i=1}^{\left|Q^{c}\right|} \frac{\left(Q_{d e p} \cdot|Q|\right)-F I_{d e g}(i)}{\left|Q^{c}\right|} . \\
C D=\frac{\max _{i=1}^{|C|}\left(R_{i} \cdot\left|Q^{i}\right|\right)}{|Q|^{2} \cdot|C|} .
\end{gathered}
$$




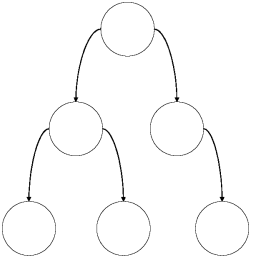

(a) Forward

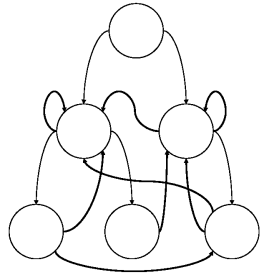

(b) Backward

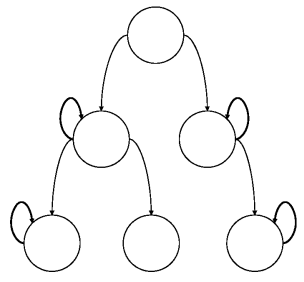

(c) Looping

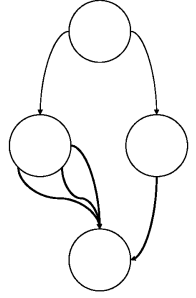

(d) Converging

Fig. 7. Examples of partial causes of state irregularity.

\subsection{State Irregularity}

Some states in DFA have a large fan-in whereas other do not. This unbalanced distribution makes DFA encoding less memory efficient. Here we first present several causes of this irregularity. Then we quantify this irregularity with the metric state irregularity. Understanding of state irregularity motivates our optimization techniques presented in Section 4.3. First we will examine a few causes of irregularity in DFA. The body of a DFA contains many forward matching transitions as shown in Figure 7(a). However, the main causes of state irregularity are backward, looping, and converging transitions as shown in Figure 7.

Backward transitions point to the beginning or middle of another pattern. These are transitions from a state on an input character that is not a valid character for the next forward matching transition. Therefore, a transition is followed for said character to a destination state that represents the longest prefix that matches the sequence of characters that match the failing state. Figure 7(b) shows these backward transitions in bold.

Looping transitions occur when a regular-expression repetition operator is used. A state or series of states will continue to loop back as long as a specific string or character occurs in the input stream. Figure 7(c) shows looping transitions in bold. Converging transitions are when a state has more than one labeled transition to another state, or, more than one state fans-in to the same state. Figure $7(\mathrm{~d})$ shows converging transitions in bold.

In order to quantify this irregularity, we will again use the fan-in degree sequence from Equation (2). If we label the largest value in $F I_{d e g}$ to be $F I_{\max }$, then we can define State Irregularity $(S R)$, by Equation (6). $S R$ is the level of irregular edge distribution. As the value of $S R$ approaches one, the more irregularity that exists relative to the states with the largest fan-in degrees. This irregularity manifests itself as wasted memory. However, an $S R$ value of zero means that each state has the exact same fanin degree, yielding better memory efficiency. The ideal scenario is if both conditions 1 and 2 from Equation (7) are met.

$$
\begin{gathered}
S R=\frac{\sum_{i=1}^{|Q|}\left(1-\frac{F I_{\text {deg }}(i)}{F I_{\max }}\right)}{|Q|} . \\
\text { 1.) } S R=0 \quad \text { 2.) } \sum_{i=1}^{|Q|} F I_{\text {deg }}(i)=|Q| .
\end{gathered}
$$


ALGORITHM 1: Algorithm for Finding State Independence for Self-Addressable Encoding and Character-Aware Encoding

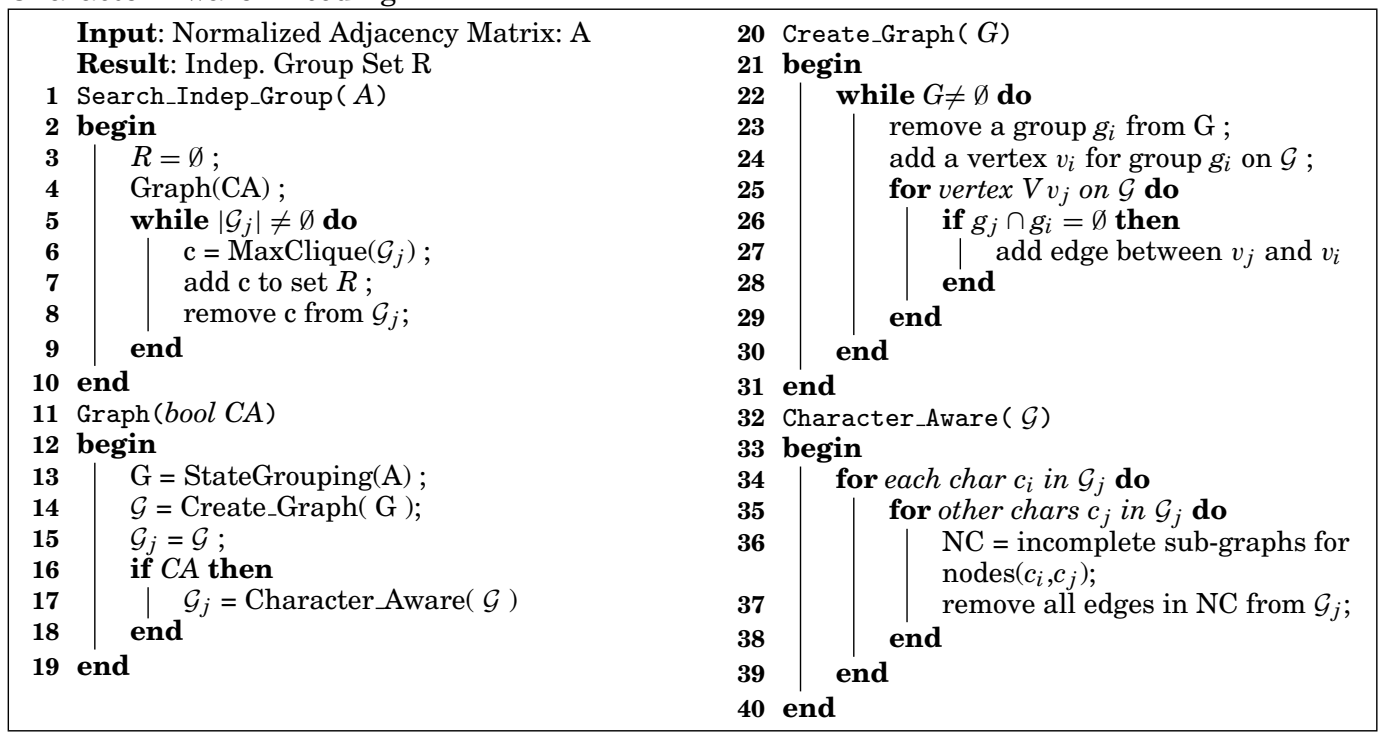

\section{ENCODING OPTIMIZATION}

To exploit the previously discussed characteristics we present three encoding optimizations: self-addressable encoding, character-aware encoding, and split-level optimization. Self-addressable encoding exploits state independence to minimize memory, character aware encoding resolves the problem of DFA lookup due to character distribution, and split-level minimizes state irregularity to enhance the effects of self-addressable encoding.

\subsection{Self-Addressable Encoding-Exploiting State Independence}

A DFA should be encoded into memory such that it is compressed as much as possible. Self-addressable encoding does this by using one code word for each state in a DFA. A code word for state $q$ contains all next-states of $q$. Each next state in the code word is stored in a fixed entry with other states that are independent according to state independence as discussed in Section 3.1. This compresses each code word substantially. There are two steps for generating self-addressable code words: identifying state independence and deriving state codes.

4.1.1. Identifying State Independence. The first step in self-addressable encoding is to find dependencies between all states. Dependencies can be quickly checked by forming a group for each state, which consists of its fan-in states. The groups for the DFA shown in Figure 8(a) are as follows, where $\mathrm{Gi}$ is the group for state $i: \mathrm{G} 1[1,3,4,5,6,8]$, G2[1,2,3,5,7,8,9], G3[1], G4[1,5], G5[2,7,9], G6[3], G7[4], G8[5], G9[6]. These groups can be easily formed by using the column for each state in the adjacency matrix as the source for its group members.

From these groups, a graph of state independence can be formed. If two groups share a common member, those two states (groups) are dependent because they share a fan-in state. In the adjacency matrix in Figure 8(b), if two columns share a one in the same row, then those states are dependent. An independence graph $G$ is then formed by connecting states without any dependency. A dependence graph could also be created 


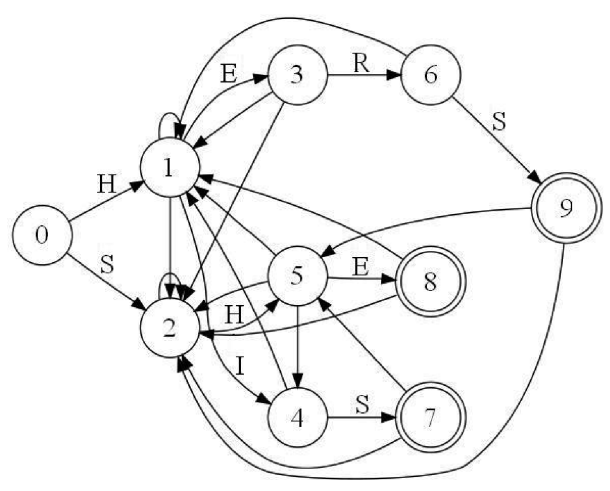

(a) DFA graph

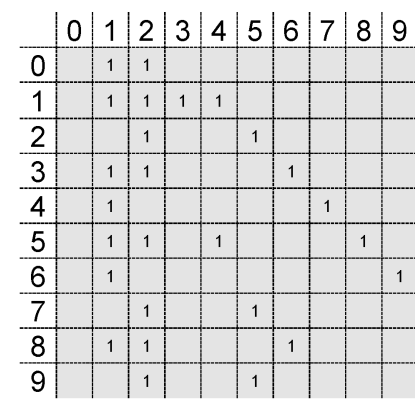

(b) DFA adjacency matrix

Fig. 8. Different representations for patterns SHE, HERS, HIS.

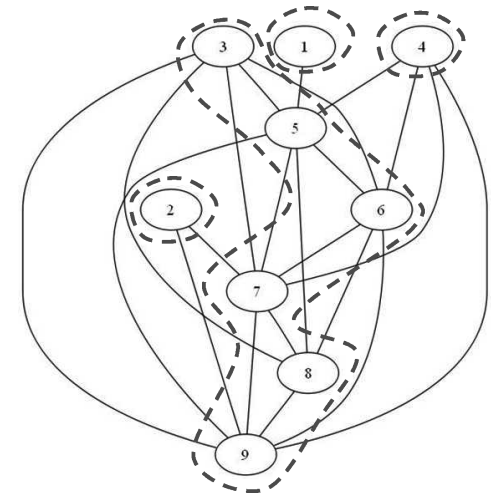

Fig. 9. State independence graph for self-addressable encoding.

Table I. Sets for State Independence

\begin{tabular}{|l|l|c|}
\hline Entry & \multicolumn{1}{|c|}{ States } & Bits \\
\hline \hline E0 & 1 & 1 \\
\hline E1 & 2 & 1 \\
\hline E2 & 356789 & 3 \\
\hline E3 & 4 & 1 \\
\hline
\end{tabular}

by taking the compliment of $G$. Figure 9 shows a completed independence graph for the DFA in Figure 8.

4.1.2. Deriving State Codes. We then search the independence graph for sets of mutually independent states. We want to locate maximal sized cliques in the independence graph, each clique forming a set of states. Each set of states will then be given an associated entry $E$ in memory and only states from that set will be allowed to be placed in entry $E$. This ensures that there is always a predefined location in memory for any next-state.

In order to form these independent sets, a well known maximum clique algorithm is applied to the independence graph. In Figure 9, the encircled areas represent the cliques that are to become the independent sets. Table I shows the final independent sets and the entry $E$ associated with each set. The table also shows the number of bits needed to represent the value of each state in the different sets. Figure 10 shows the final locations where next-state values will be stored in memory. Each state has a 


\begin{tabular}{|c|c|c|c|c|}
\hline & $\mathrm{E}_{0}$ & $E_{1}$ & $E_{2}$ & $E_{3}$ \\
\hline$L_{q 0}$ & $q_{1}$ & $q_{2}$ & & \\
\hline$L_{q 1}$ & $\mathrm{q}_{1}$ & $\mathrm{q}_{2}$ & $q_{3}$ & $\mathrm{q}_{4}$ \\
\hline $\mathrm{L}_{\mathrm{q} 2}$ & & $\mathrm{q}_{2}$ & & \\
\hline $\mathrm{L}_{\mathrm{q} 3}$ & $q_{1}$ & $\mathrm{q}_{2}$ & $q_{6}$ & \\
\hline $\mathrm{L}_{\mathrm{q} 4}$ & $q_{1}$ & & $q_{7}$ & \\
\hline $\mathrm{L}_{\mathrm{q} 5}$ & $q_{1}$ & $\mathrm{q}_{2}$ & $q_{8}$ & $q_{4}$ \\
\hline$L_{q 6}$ & $q_{1}$ & & $q_{9}$ & \\
\hline $\mathrm{L}_{\mathrm{q} 7}$ & & $q_{2}$ & $q_{5}$ & \\
\hline$L_{q 8}$ & $q_{1}$ & $q_{2}$ & & \\
\hline$L_{q 9}$ & & $\mathrm{q}_{2}$ & $\mathrm{q}_{5}$ & \\
\hline
\end{tabular}

Fig. 10. Memory content for self-addressable coding.

Table II. State Signatures for Self-Addressable Encoding

\begin{tabular}{|c|c|}
\hline State & Signature \\
\hline \hline $\mathrm{q} 1$ & 1 \\
\hline $\mathrm{q} 2$ & 1 \\
\hline $\mathrm{q} 3$ & 001 \\
\hline $\mathrm{q} 4$ & 1 \\
\hline $\mathrm{q} 5$ & 010 \\
\hline $\mathrm{q} 6$ & 011 \\
\hline $\mathrm{q} 7$ & 100 \\
\hline $\mathrm{q} 8$ & 101 \\
\hline $\mathrm{q} 9$ & 110 \\
\hline
\end{tabular}

Table III. State Codes for Self-Addressable Encoding

\begin{tabular}{|c|l||c|c|c|c|}
\hline \multirow{2}{*}{} & \multicolumn{1}{|c||}{} & \multicolumn{4}{c|}{ State Codes } \\
\cline { 3 - 6 } & & E0 & E1 & E2 & E3 \\
\hline \hline q0 & q1 q2 & 1 & 1 & 000 & 0 \\
\hline q1 & q1 q2 q3 q4 & 1 & 1 & 001 & 1 \\
\hline q2 & q2 q5 & 0 & 1 & 000 & 0 \\
\hline q3 & q1 q2 q6 & 1 & 1 & 011 & 0 \\
\hline q4 & q1 q7 & 1 & 0 & 100 & 0 \\
\hline q5 & q1 q2 q4 q8 & 1 & 1 & 101 & 1 \\
\hline q6 & q1 q9 & 1 & 0 & 110 & 0 \\
\hline q7 & q2 q5 & 0 & 1 & 010 & 0 \\
\hline q8 & q1 q2 & 1 & 1 & 000 & 0 \\
\hline q9 & q2 q5 & 0 & 1 & 010 & 0 \\
\hline
\end{tabular}

signature code that will be stored in the entries shown in Figure 10. These signatures are shown in Table II. The signatures are assigned sequentially relative to the entry of each state starting with 1 . The final state code for a state $q$ is simply the concatenation of the signatures of the next states for $q$. The final state codes for self-addressable encoding are shown in Table III.

Algorithm 3.3 (lines 1-31) shows the detailed steps to identify state independence to generate self-addressable encoding. Self-addressable encoding minimizes memory in two ways. First, it reduces the required number of entries in state codes. Second, the values of the states can be assigned relative to their independent set, rather than relative to $Q$. For example, if a state is a member of set $S$, then the the value of that state 
Table IV. Number of Bits Required for States in Each Entry With and Without Independence

\begin{tabular}{|l|c|c|c|c|c|}
\hline Memory Implementation & E0 & E1 & E2 & E3 & Total \\
\hline \hline With Independence & 1 & 1 & 3 & 1 & 6 \\
\hline Without Independence & 4 & 4 & 4 & 4 & 16 \\
\hline
\end{tabular}

Table V. Sets for Character Independence

\begin{tabular}{|l|c|l|c|}
\hline Entry & Chars & States & Bits \\
\hline \hline E0 & H & 15 & 2 \\
\hline E1 & S & 279 & 2 \\
\hline E2 & E,R & 386 & 2 \\
\hline E3 & I & 4 & 1 \\
\hline
\end{tabular}

in memory requires $\log _{2}|S|$ bits rather than $\log _{2}|Q|$ bits. Table IV shows the number of bits reduced by leveraging state independence.

\subsection{Character-Aware Encoding-Exploiting Character Distribution}

In addition to encoding DFA with compression, our character-aware encoding allows quick isolation of the next state from the current state code. Character-aware encoding guarantees that all states that receive an incoming transition on the same character are stored in the same entry. If all states that receive transitions on the same character are guaranteed to be stored in the same entry $E$ in memory, then only one entry in memory need be checked to find the next state. In other words, if the input character is $c$ and the current state is $q_{i}$, the engine only needs to check one entry of the code word for $q_{i}$ in order to obtain the next state for $q_{i}$. Instead of deriving state independence, for character-aware we need to find character independence. Algorithm 3.3 (lines 1-40) shows how to derive state and character independence.

4.2.1. Identifying Character Independence. Two characters are independent if all states that receive transitions on those characters are mutually independent. Identifying character independence begins the same as identifying state independence. Dependencies are found between states, and groups are formed, however these groups can be simplified to the character for any state rather than the state itself. Combining members for groups of like characters is simply the union of those two groups: $G^{C_{k}} S_{i} \cup G^{C_{k}} S_{j}$. The groups for the DFA in Figure 8(a) are as follows: $\mathrm{H}[1,2,3,4,5,6,7,8,9]$, S[1,2,3,4,5,6,7,8,9], $\mathrm{E}[1,5], \mathrm{I}[1,5], \mathrm{R}[3]$.

4.2.2. Deriving State Codes. We then find maximum cliques from a character independence graph as shown in Figure 11. Table V shows the final independent sets and the entry $E$ associated with each set. The table also shows the number of bits needed to represent the value of each state in the different sets. Also in Table V are the characters of all states in any given entry. For example, for entry $E_{0}$, all states in that set are ones that receive character $\mathrm{H}$ transitions.

If a state has two incoming transitions on two characters such as in the regular expression '|' operation, then said state is associated with both characters when deriving character independence. If the two characters are independent, then states of those characters are stored in the same entry. If the two characters are dependent then states of the two different characters are stored in different entries, and the state that receives transitions on both characters is replicated between the two entries.

Figure 12 shows the final locations where next-state values will be stored in memory. Each state has a signature code that will be stored in the entries shown in Figure 10. These signatures are shown in Table VI. The signatures are assigned sequentially relative to the entry of each state starting with 1 . In addition, each next state entry 


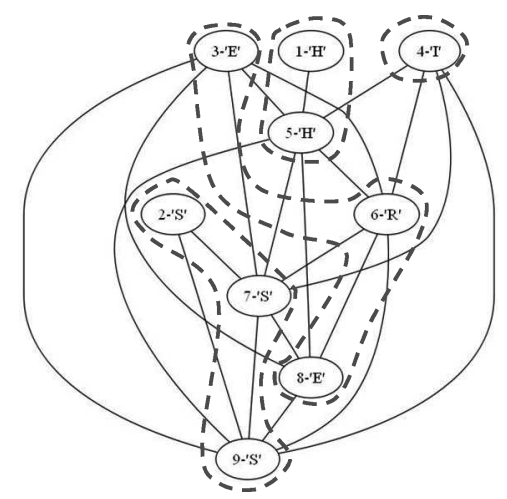

Fig. 11. Character independence graph.

\begin{tabular}{|c|c|c|c|c|}
\hline & $\mathrm{E}_{0}$ & $E_{1}$ & $E_{2}$ & $E_{3}$ \\
\hline $\mathrm{L}_{\mathrm{q} 0}$ & $\mathrm{q}_{1}$ & $\mathrm{q}_{2}$ & & \\
\hline$L_{q 1}$ & $q_{1}$ & $\mathrm{q}_{2}$ & $\mathrm{q}_{3}$ & $\mathrm{q}_{4}$ \\
\hline $\mathrm{L}_{\mathrm{q} 2}$ & & $\mathrm{q}_{2}$ & & \\
\hline$L_{q 3}$ & $q_{1}$ & $\mathrm{q}_{2}$ & $\mathrm{q}_{6}$ & \\
\hline $\mathrm{L}_{\mathrm{q} 4}$ & $q_{1}$ & $q_{7}$ & & \\
\hline $\mathrm{L}_{\mathrm{q} 5}$ & $\mathrm{q}_{1}$ & $\mathrm{q}_{2}$ & $\mathrm{q}_{8}$ & $q_{4}$ \\
\hline $\mathrm{L}_{q 6}$ & $q_{1}$ & $\mathrm{q}_{9}$ & & \\
\hline$L_{q 7}$ & $\mathrm{q}_{5}$ & $\mathrm{q}_{2}$ & & \\
\hline $\mathrm{L}_{\mathrm{q} 8}$ & $\mathrm{q}_{1}$ & $\mathrm{q}_{2}$ & & \\
\hline$L_{q 9}$ & $q_{5}$ & $\mathrm{q}_{2}$ & & \\
\hline
\end{tabular}

Fig. 12. Memory content for character aware coding.

Table VI. State Signatures for Character-Aware Encoding

\begin{tabular}{|c|c|}
\hline State & Signature \\
\hline \hline $\mathrm{q} 1$ & 01 \\
\hline $\mathrm{q} 5$ & 10 \\
\hline $\mathrm{q} 2$ & 01 \\
\hline $\mathrm{q} 7$ & 10 \\
\hline $\mathrm{q} 9$ & 11 \\
\hline $\mathrm{q} 3$ & 01 \\
\hline $\mathrm{q} 8$ & 10 \\
\hline $\mathrm{q} 6$ & 11 \\
\hline $\mathrm{q} 4$ & 1 \\
\hline
\end{tabular}

has a corresponding character signature, as shown in Table VII. The final state code for a state $q$ is simply the concatenation of the signatures of the next states for $q$ and their corresponding character signatures. The final state codes for character-aware encoding are shown in Table VIII.

\subsection{Split-Level-Exploiting State Irregularity}

Split-level optimization is necessary in order to reduce irregularity and maximize state and character independence. It removes many transitions in order to reduce irregularity. Figure 13(a) shows a DFA for several patterns. There are a substantial 
Table VII. Character Signatures and Entries for Character-Aware Encoding

\begin{tabular}{|l|c|c|}
\hline Char & Signature & Entry \\
\hline \hline H & 0 & 0 \\
\hline S & 0 & 1 \\
\hline E & 0 & 2 \\
\hline R & 1 & 2 \\
\hline I & 0 & 3 \\
\hline
\end{tabular}

Table VIII. State Codes for Character-Aware Encoding. C Stands for Character Signature and S Stands for State Signature

\begin{tabular}{|c|c|c|c|c|c|c|c|c|c|}
\hline \multirow[b]{3}{*}{ State } & \multirow[b]{3}{*}{ Next States } & \multicolumn{8}{|c|}{ State Codes } \\
\hline & & \multicolumn{2}{|c|}{ E0 } & \multicolumn{2}{|c|}{ E1 } & \multicolumn{2}{|c|}{ E2 } & \multicolumn{2}{|c|}{ E3 } \\
\hline & & $\mathrm{C}$ & $\mathrm{S}$ & $\mathrm{C}$ & S & $\mathrm{C}$ & $\mathrm{S}$ & $\mathrm{C}$ & $\mathrm{S}$ \\
\hline$\overline{q q 0}$ & $\bar{q}$ q1q2 & 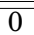 & 01 & $\overline{00}$ & 01 & 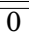 & 00 & 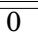 & 0 \\
\hline$q 1$ & q1 q2 q3 q4 & 0 & 01 & 0 & 01 & 0 & 01 & 0 & 1 \\
\hline $\mathrm{q} 2$ & q2 q5 & 0 & 10 & 0 & 01 & 0 & 00 & 0 & 0 \\
\hline$q^{3}$ & q1 q2 q6 & 0 & 01 & 0 & 01 & 1 & 00 & 0 & 0 \\
\hline $\mathrm{q} 4$ & q1 q7 & 0 & 01 & 0 & 10 & 0 & 00 & 0 & 0 \\
\hline q5 & q1 q2 q4 q8 & 0 & 01 & 0 & 01 & 0 & 10 & 0 & 1 \\
\hline$q 6$ & q1 q9 & 0 & 01 & 0 & 11 & 0 & 00 & 0 & 0 \\
\hline q7 & q2 q5 & 0 & 10 & 0 & 01 & 0 & 00 & 0 & 0 \\
\hline q8 & q1 q2 & 0 & 01 & 0 & 01 & 0 & 00 & 0 & 0 \\
\hline q9 & q2 q5 & 0 & 10 & 0 & 01 & 0 & 00 & 0 & 0 \\
\hline
\end{tabular}

amount of transitions causing irregularity, most of which are backward transitions. We remove these transitions and replace them with a lookup between levels of the DFA. We split the DFA into either two or three levels. A two-level DFA, we call single split-level, and a three-level DFA, we call multiple split-level. The end result of this optimization is a DFA with fewer transitions that can be more efficiently encoded using our encoding techniques.

4.3.1. Single Split-Level. For any state that receives more than one transition, a single transition can be retained as a lookup transition to that state. The remaining transitions to that state can subsequently be removed. For example, in Figure 13(a), state 1 receives many backward transitions. These transitions always occur on the letter "a." If the transition $\delta\left(q_{0}\right.$, " $a$ ") $\rightarrow q_{1}$ is used as a lookup transition, then a one-to-many relationship is created between all transitions to state 1 and the lookup transition. The same can be done for all transitions $\delta\left(q_{0}, c_{j}\right) \rightarrow q_{j}$, yielding the DFA in Figure 13(b). If a state fails for any character, the lookup transitions can be used to determine the appropriate next-state.

4.3.2. Multiple Split-Level. After one level of split-level is applied as in Figure 13(b), nonforward matching transitions are still present. These transitions can still contribute a great deal to memory inefficiency, as well as matching operation complexity. Complete split-level can be achieved by adding another level of the split-level optimization. After completing this split-level, as seen in Figure 13(c), each lookup block is completely regular relative to itself. When storing $\delta$ in memory for a normalized DFA, there is little on no wasted memory due to state irregularity.

\section{MEMORY-BASED ENGINES}

This section presents three memory-based DFA implementations that exploit the characteristics and optimizations discussed in previous sections. First, we present the general architecture of the memory-based pattern matching engine in Section 5.1. Then three specific implementations are introduced in Sections 5.2, 5.3, and 5.4. These 


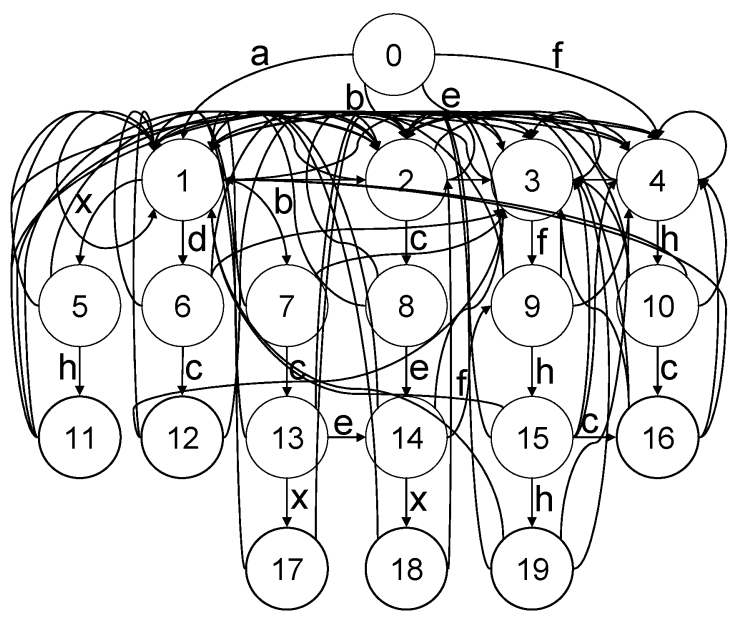

(a) No split-level

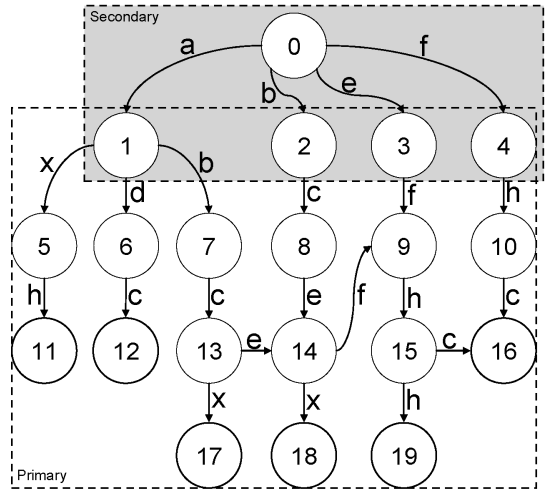

(b) One level split

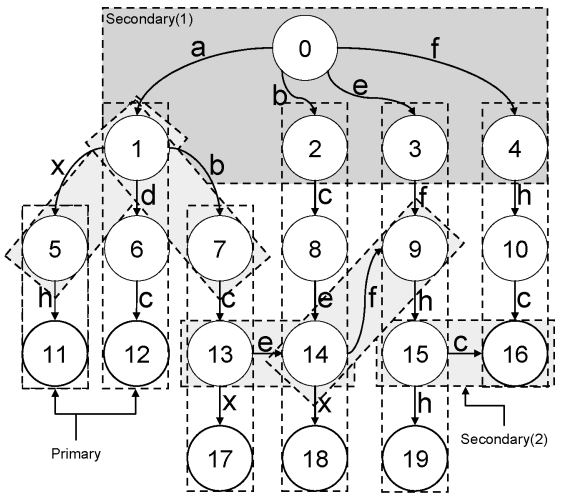

(c) Two level split

Fig. 13. DFAs for Patterns (axh, adc, abcx, bcex, efhh, fhc) with varying split-level.

engines leverage different techniques and demonstrate the tradeoffs between memory requirements and performance.

\subsection{General Engine Architecture}

The general architecture for the memory-based pattern matching engines is shown in Figure 14. From this figure it can be seen that each state has a code word in memory with up to $k$ next-state entries. Given a current state (c.s.) stored in the state register $S R$, and an input character stored in the character register $C R$, an algorithm specific to the engine is used to search the code word of the c.s. and find the n.s. entry in memory. The differences in the engines presented in the next section are in the methods used to store the code words for each state in memory, as well as the algorithm used to isolate the appropriate next state.

In the following three sections, we encode the DFA from Figure 8 using three different engines. We then give an an example of the operation of each engine using a uniform example. Figure 16 shows the sample transition used for the examples. The current state (c.s.) is $q_{1}$ and the input character is "I," making the next state (n.s.) $q_{4}$. 


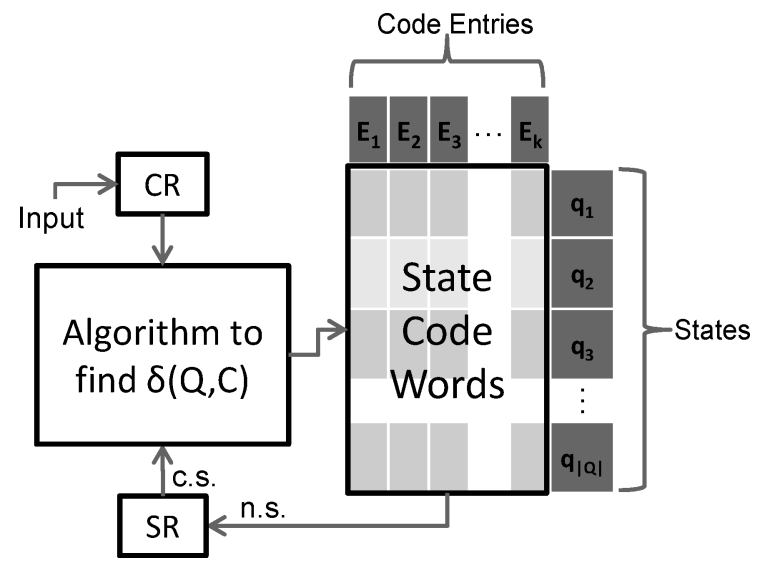

Fig. 14. Memory-based engine, general architecture.

\subsection{Self Addressable Memory-Based Finite State Machine}

SAM-FSM Architecture. Self Addressable Memory-Based Finite State Machine (SAMFSM) encodes a DFA using self-addressable encoding. Its architecture is shown in Figure 15(a). It examines all the entries in the current state code in order to isolate the next state. This operation must either be performed sequentially or in parallel.

SAM-FSM Operating Example. An example of the operation of SAM-FSM is shown in Figure 17. This example encodes the DFA in Figure 8. It follows a single transition from state $q_{1}$ to state $q_{4}$ on input character "I," as shown in Figure 16. SAM-FSM begins by checking the first entry of the code for $q_{1}$ against the input character "I." If there is a match, it adds the value in that entry to the offset for that entry to find the address of the next state. If there is not a match, it moves to the next entry and repeats the process.

\subsection{Portable Predictive Pattern Matching Finite State Machine}

$P^{3}$ FSM Architecture. The portable Predictive Pattern Matching Finite State Machine $\left(P^{3} F S M\right)$ first applies the single split-level DFA optimization, then encodes the DFA using character-aware encoding. The architecture for $P^{3} F S M$ is shown in Figure 15(b). Because $P^{3} F S M$ uses character-aware encoding, the placement of states into entries is limited by the character distribution in a DFA. This means that the compression level of $P^{3} F S M$ is not as high as simply using self-addressable encoding. Character-aware encoding however guarantees that all states that receive an incoming transition on the same character are stored in the same entry. This means that given an input character $c, P^{3}$ FSM knows what entry to check in the current state code to find the next state for character $c$. So only one entry of the current state code must be examined in order to isolate the next state.

$P^{3} F S M$ Operating Example. Figure 18 shows an example of $P^{3} F S M$ that encodes the DFA in Figure 8. It illustrates a transition from state $q_{1}$ to state $q_{4}$ on input character "I," as in Figure 16. $P^{3} F S M$ begins by checking the secondary memory for the entry for character "I". This entry is then examined in the code of $q_{1}$ in primary memory to see if it contains character "I." If it finds a match it adds the value from primary memory to the offset for character "I" in order to find the address of the next state in primary memory. 


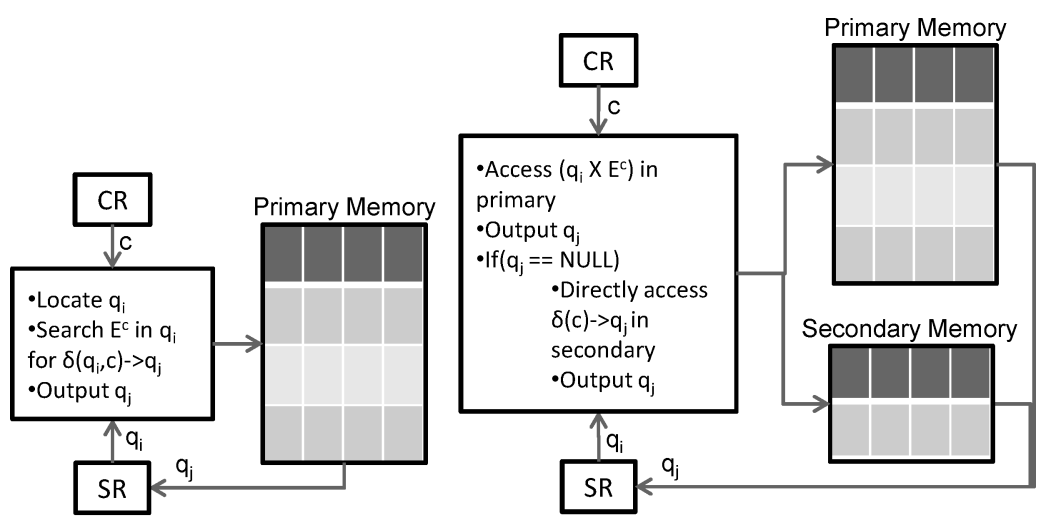

(a) Architecture for SAM-FSM

(b) Architecture for P3FSM

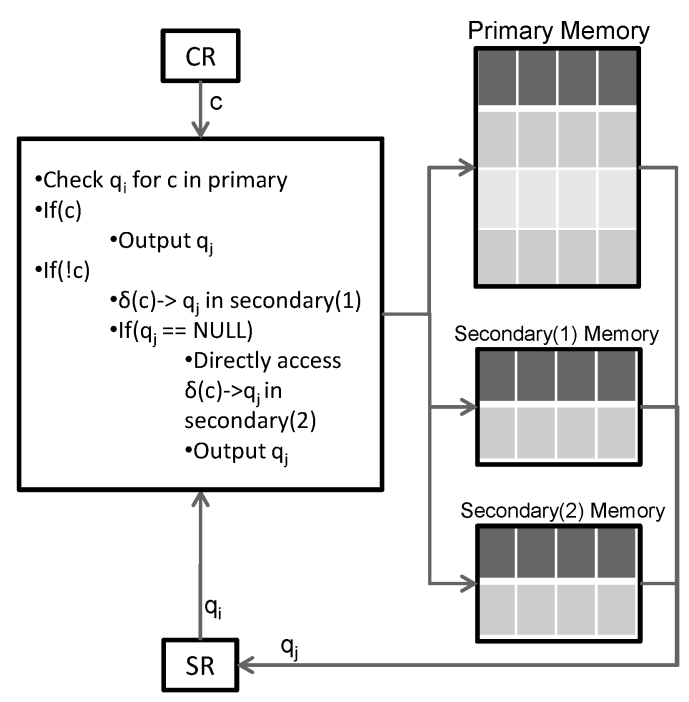

(c) Architecture for SI-FSM

Fig. 15. Memory-based pattern matching engines.

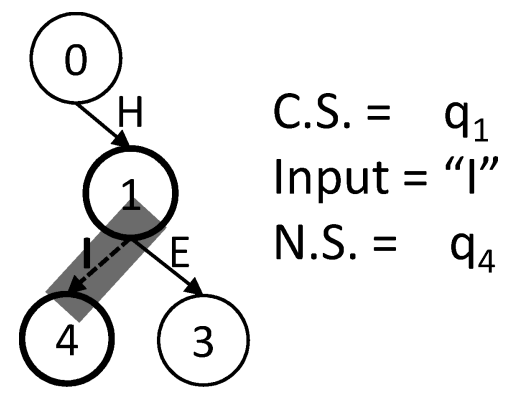

Fig. 16. Sample transition used to demonstrate engine operation. This is a partial section of the DFA in Figure 8, a transition from state $q_{1}$ to $q_{4}$ on input "I." 


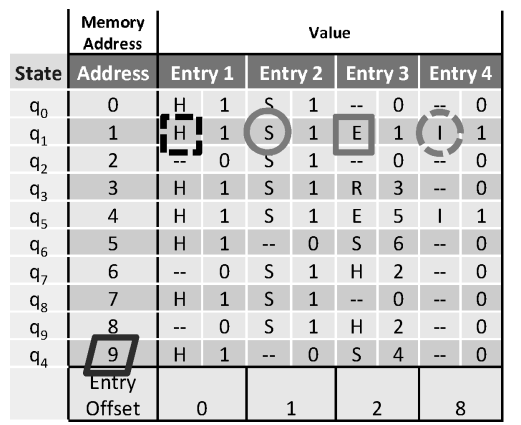

$$
\begin{aligned}
& \text { Step 1: Compare 'I' with character Entry } 1 \\
& \text { Result: } H \neq 1 \\
& \text { Step 2: } \\
& \text { Sempare 'I' with character Entry 2 } \\
& \text { Result: } S \neq 1 \\
& \text { Step 3: Compare 'I' with character Entry 3 } \\
& \text { Result: } E \neq 1 \\
& \text { Step 4: Compare 'I' with character Entry 4 } \\
& \text { Result: } I=1 \text {, Success } \\
& \text { Step 5: Calculate N.S. Address w/Entry offset } \\
& \text { Result: Address }=1+8=9 \\
& \text { N.S. }=\mathbf{q}_{4}
\end{aligned}
$$

Fig. 17. Example operation of SAM-FSM engine. Transition from state $q_{1}$ to $q_{4}$ on input "I"from Figure 16
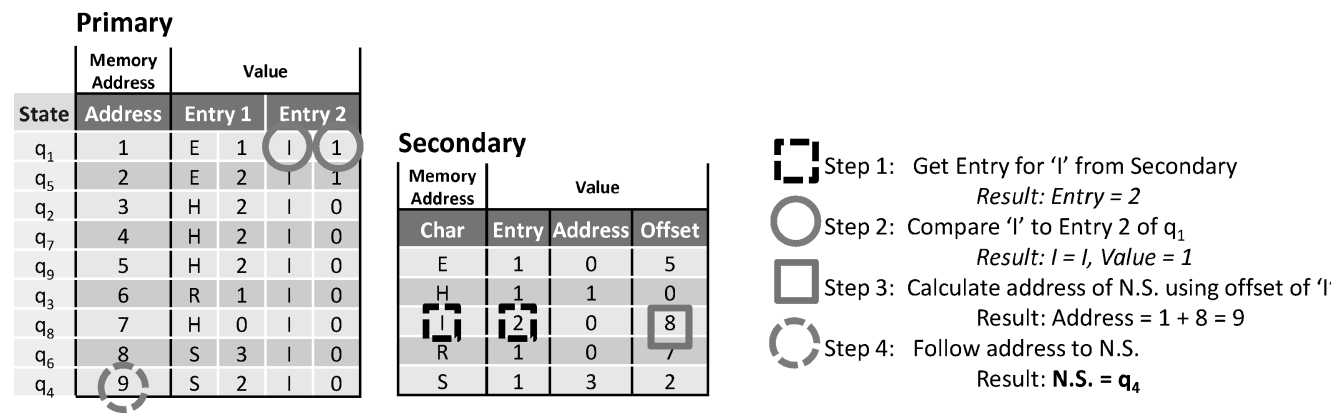

Fig. 18. Example operation of $P^{3} F S M$ engine. Transition from state $q_{1}$ to $q_{4}$ on input "I" from Figure 16 .
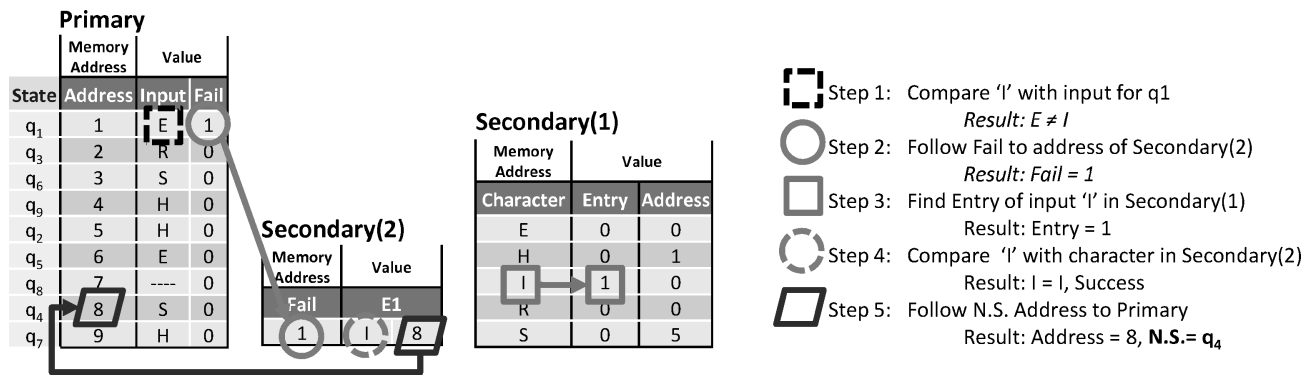

Fig. 19. Example operation of SI-FSM engine. Transition from state $q_{1}$ to state $q_{4}$ on input "I" from Figure 16.

\subsection{Simple Instruction Finite State Machine}

SI-FSM Architecture. Simple Instruction Finite State Machine (SI-FSM) first applies the multiple split-level DFA optimization, then encodes the DFA using character-aware encoding. The architecture for SI-FSM is shown in Figure 15(c). SI-FSM uses a threememory table architecture to store the transitions of a multiple split-level optimized DFA. This allows it to achieve higher compression than $P^{3} F S M$, while still using character-aware encoding. However, this three-table architecture means that SI-FSM occasionally has to lookup to a third table and therefore achieves slightly lower performance than $P^{3} F S M$.

SI-FSM Operating Example. Figure 19 shows an example of SI-FSM that encodes the DFA in Figure 8. It illustrates a transition from state $q_{1}$ to state $q_{4}$ on input character "I," as in Figure 16. As the current state is $q_{1}$, SI-FSM begins by comparing the input "I" to the input field of the primary memory code for $q_{1}$. On failure, SI-FSM uses the 
"Fail" field to identify the address of the appropriate code in the secondary(2) memory. It also looks up the entry for the character "I" in the secondary(1) memory table. It then checks the entry in the secondary $(2)$ memory table against the input character "I." It uses the value from the secondary(2) memory as the address of the next state in primary memory.

\section{RESULTS}

In this article thus far, we have presented three metrics that characterize DFA in terms of memory and performance. These metrics are state independence (SD), character distribution (CD), and state irregularity (SR). We have also presented three encoding optimization techniques that address the issues characterized by our three metrics. Self-addressable encoding exploits state independence to reduce memory, characteraware solves the problems of DFA lookup, and split-level reduces state irregularity and in turn increases state independence to ultimately reduce memory. To demonstrate these encoding optimization techniques, we have presented three complete memorybased pattern matching engines: SAM-FSM, $P^{3} F S M$, and SI-FSM.

In this section we perform analysis and present experimental results related to our three metrics, encoding optimizations and engines. Section 6.1 presents different DFA and the properties of DFA that affect our three metrics. This section also discusses the effect of our metrics on memory and performance. Section 6.2 evaluates our encoding optimizations along with other optimizations, which demonstrates how the optimizations used by our engines affect memory and performance. Section 6.3 compares our pattern-matching engines, along with other engines in terms of memory and performance.

\subsection{DFA Characterization Metrics Evaluation}

Table IX shows the SD, SR and CD metric values for three different DFA. The DFA utilized to derive the metric values in Table IX are shown in Figure 20. The value of SD for the DFA in Figure 20(a) is 1.0. Since the SD metric approximates the percentage of memory caused by dependency between states, it can be assumed from this value of 1.0 that the dependency in Figure 20(a) is 100\%. Each state in Figure 20(a) fans in to every state in the DFA. This means that every state in the DFA is dependent with every other state. The SD value for Figure 20(b) is 0.250 . In this DFA, state 1 has a dependency with states 2 through 5 . However states 2 through 5 are all independent of all other states. SD is calculated as $\frac{1}{\log _{2}(5)+1}$, where the 1 is the dependent states and the 5 is the independent states. The result of this is $\frac{1}{4}$, or 0.250 .

The SR value for the DFA in Figure 20(a) is zero. SR is the metric that evaluates the amount of irregularity in DFA, therefore the value for SR is zero because the DFA is completely regular. Each nonzero state in the DFA in Figure 20(a) has the same $F I_{\text {deg }}$ as every other state, making the DFA completely regular. Even if a DFA is completely regular, it does not necessarily mean that the implementation of that DFA will be memory-efficient. In this case, even though $\mathrm{SR}=0$, the value of $\mathrm{SD}=1$, meaning the DFA contains no independence. The value of CD for the DFA in Figure 20(a) is 0.166. This value is the number of lookups per character, relative to the size of the DFA. It is obvious from Figure 20(a) that the required number of lookups for any character will only be one because $|Q| \approx|C|$.

Table $\mathrm{X}$ shows the values of the three metrics for a group of DFAs built from different Snort [2009] rulesets. The table shows the number of patterns, number of characters, size of the character set, and number of states for each DFA. One clear trend observed from Table $\mathrm{X}$ is that as the size of the DFA increases, so does SD. This can be accounted for by the following explanation. As the number of patterns increase, the dependence 
Table IX. DFA Characterized by SD, CD and SR

\begin{tabular}{|l||c|c|c||c|c|c|}
\hline & & $\begin{array}{c}\text { \# Unique } \\
\text { Characters } \\
|C|\end{array}$ & $\begin{array}{c}\text { \# States } \\
|Q|\end{array}$ & $\begin{array}{c}\text { State } \\
\text { Independence } \\
\text { (SD) }\end{array}$ & $\begin{array}{c}\text { Character } \\
\text { Distribution } \\
\text { (CD) }\end{array}$ & $\begin{array}{c}\text { State } \\
\text { Irregularity } \\
\text { (SR) }\end{array}$ \\
\hline \hline Patterns(a) & 6 & 6 & 7 & 1.000 & 0.166 & 0.000 \\
\hline Patterns(b) & 1 & 6 & 7 & 0.250 & 0.051 & 0.881 \\
\hline Patterns(c) & 2 & 2 & 7 & 0.500 & 0.486 & 0.583 \\
\hline
\end{tabular}

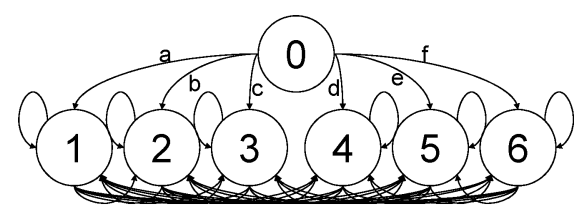

(a) DFA for patterns $\{a, b, c, d, e, f\}$

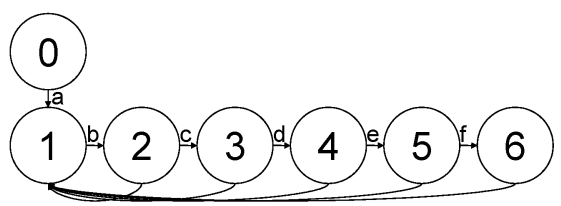

(b) DFA for pattern $\{$ abcdef $\}$

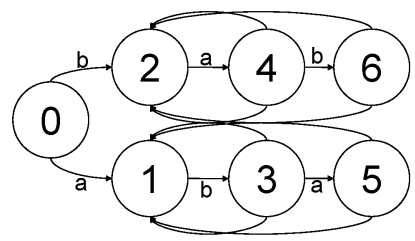

(c) DFA for patterns $\{a b a, b a b\}$

Fig. 20. Examples illustrating varying DFA metrics in Table IX.

Table X. Group of Snort Ruleset Characteristics

\begin{tabular}{|l||c|c|c|c||c|c|c|}
\hline & \# Patterns & \# Characters & $\begin{array}{c}\text { \# Unique } \\
\text { Characters } \\
|C|\end{array}$ & $\begin{array}{c}\text { \# States } \\
|Q|\end{array}$ & $\begin{array}{c}\text { State } \\
\text { Independence } \\
\text { (SD) }\end{array}$ & $\begin{array}{c}\text { Character } \\
\text { Distribution } \\
(\text { CD) }\end{array}$ & $\begin{array}{c}\text { State } \\
\text { Irregularity } \\
(\text { SR })\end{array}$ \\
\hline Synthetic 1 & 1 & 20 & 13 & 20 & 0.286 & 0.024 & 0.815 \\
\hline Synthetic 2 & 2 & 37 & 19 & 37 & 0.333 & 0.021 & 0.885 \\
\hline Synthetic 3 & 5 & 111 & 32 & 93 & 0.462 & 0.018 & 0.918 \\
\hline Synthetic 4 & 20 & 363 & 55 & 302 & 0.591 & 0.022 & 0.921 \\
\hline IMAP & 33 & 232 & 34 & 181 & 0.619 & 0.041 & 0.936 \\
\hline FTP & 58 & 343 & 41 & 296 & 0.700 & 0.061 & 0.942 \\
\hline Policy & 88 & 1009 & 49 & 875 & 0.767 & 0.057 & 0.963 \\
\hline Web-CGI & 200 & 2361 & 42 & 1733 & 0.773 & 0.040 & 0.989 \\
\hline
\end{tabular}

between states tends to follow. This is because, since each new pattern that does not share a prefix with another pattern, all states currently in the DFA will form backward transitions to the first character prefix of the new pattern. This causes a nonlinear increase in the amount of transitions in the DFA as more patterns are added. This means that rather than dependence staying constant with each added pattern, it increases, which in turn increases SD.

The same concept affects the value of SR as well. If a pattern $P$ is added to a DFA and the number of states before adding pattern $P$ is $|Q|$, then the number of transitions added to the DFA can be approximated as $(P$.length $\cdot|Q|+1)+|Q|$. This nonlinear increase in transitions causes irregularity to increase with the number of patterns as well: hence the increase in SR seen in Table X.

A less consistent trend can be seen for CD in Table X. This trend is not as strong as that for SD and SR. The reason for this is that CD depends not only on transitions, but also on how characters are distributed from the characters set $|C|$. A lopsided 


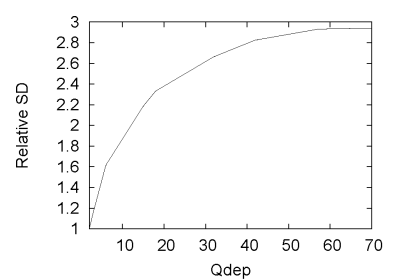

(a) SD vs. Qdep

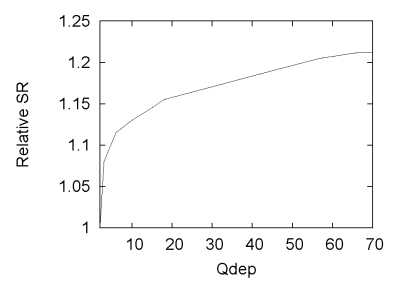

(b) SR vs. Qdep

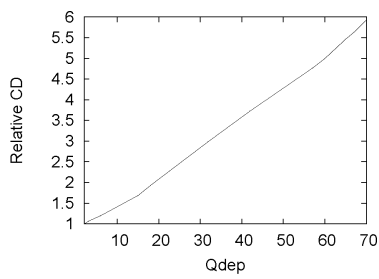

(c) CD vs. Qdep

Fig. 21. Number of dependent states affecting DFA characteristics. $Q_{d e p}$ is defined as the average number of fan-in transitions per state.

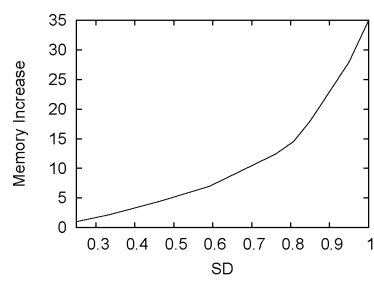

(a) Memory increases with State dependence(SD)

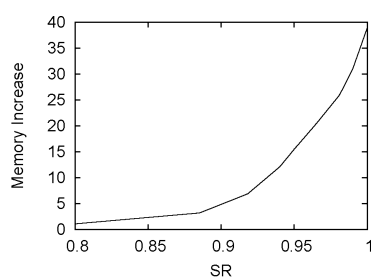

(b) Memory increases with State irregularity(SR)

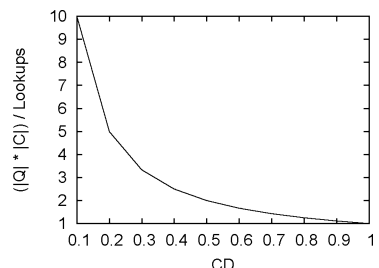

$\mathrm{CD}$

(c) Performance decreases with Character distribution(CD)

Fig. 22. Memory and performance changing with metric values. As the values of SD and SR increase, the memory requirement increases. As the value of $\mathrm{CD}$ increases, performance suffers.

distribution of characters tends to produce a larger value for CD than a uniform distribution. The increase in CD can be attributed to the increase in the size of $|C|$.

Figure 21(a) shows the increase in SD as the value of $Q_{\text {dep }}$ increases. This shows the expected trend from the relationship between $\mathrm{SD}$ and $Q_{d e p}$, as expressed by Equation (1). SR also increases with $Q_{\text {dep }}$, as shown in Figure 21(b). The approximate transition increase calculated by $(P . l e n g t h \cdot|Q|+1)+|Q|$, also causes an increase in $Q_{\text {dep }}$ so it is only natural that we see this trend between SR and $Q_{\text {dep }}$.

As $Q_{d e p}$ increases, so does CD. This is due to the increase in the number of entries needed to store each next-state in memory. More entries in memory need to be searched for each character as $Q_{\text {dep }}$ increases, and therefore, CD increases with $Q_{d e p}$. This is shown in Figure 21(c). The relationship of CD to $Q_{d e p}$ in this range of values of $Q_{d e p}$ is near linear.

Figure 22 shows the effects of the metrics on memory and performance. Assuming states are stored with a self-addressable encoding according to their independence, an increase in memory will be seen as SD increases, as observed in Figure 22(a). The same can be said for SR as observed in Figure 22(b). Figure 22(c) shows the ratio of the product of $|Q| \cdot|C|$ to the number of lookups as it relates to $\mathrm{CD}$. The higher the value of $\mathrm{CD}$, the lower this ratio becomes, and therefore the larger the number of lookups required for each character, relative to the size of the DFA.

\subsection{State Encoding Techniques}

Table XI shows the values of SD, CD, and SR for different DFA with and without the split-level optimization. The table also presents the maximum number of transitions that must be followed in order to process a single character using a particular optimized DFA.

The unoptimized DFA retain high values for SD and SR, as well as CD. These are the typical values for the metrics as observed in Section 6.1. However, in a DFA, no more 
Table XI. Split-Level Optimization Affecting the Metric Values

\begin{tabular}{|c|c|c|c|c|c|}
\hline Optimization & Ruleset & SD & CD & SR & Max Lookup \\
\hline \multirow{4}{*}{ DFA (unoptimized) } & IMAP & 0.619 & 0.041 & 0.936 & \multirow{4}{*}{1} \\
\hline & FTP & 0.700 & 0.061 & 0.942 & \\
\hline & Policy & 0.767 & 0.057 & 0.963 & \\
\hline & Web-CGI & 0.773 & 0.040 & 0.989 & \\
\hline \multirow{4}{*}{ Split-level (Single level) } & IMAP & 0.111 & 0.003 & 0.026 & \multirow{4}{*}{2} \\
\hline & FTP & 0.100 & 0.003 & 0.013 & \\
\hline & Policy & 0.091 & 0.002 & 0.006 & \\
\hline & Web-CGI & 0.083 & 0.002 & 0.005 & \\
\hline \multirow{4}{*}{ Split-level (Multi level) } & IMAP & 0.006 & 0.003 & 0.002 & \multirow{4}{*}{3} \\
\hline & FTP & 0.003 & 0.003 & 0.001 & \\
\hline & Policy & 0.001 & 0.002 & 0.000 & \\
\hline & Web-CGI & 0.001 & 0.002 & 0.000 & \\
\hline
\end{tabular}

Table XII. Regular Expression Rulesets from Snort. SD and SR Metrics for a Character-Aware Encoding of the DFA

\begin{tabular}{|l|c|c|c|c|c|c|}
\hline & & & \multicolumn{2}{|c|}{ Split-Level } & \multicolumn{2}{c|}{ D2FA } \\
\hline Ruleset & \# Regex & \# States & SD & SR & SD & SR \\
\hline \hline POP3 & 13 & 97 & 0.125 & 0.294 & 0.101 & 0.211 \\
\hline SMTP & 14 & 82 & 0.125 & 0.319 & 0.105 & 0.219 \\
\hline Exploit & 21 & 1657 & 0.329 & 0.417 & 0.192 & 0.352 \\
\hline VOIP & 39 & 3740 & 0.311 & 0.506 & 0.185 & 0.443 \\
\hline
\end{tabular}

than one transition ever needs to be followed in order to find the appropriate next state for any given current state. This is the benefit of an unoptimized DFA. This does not necessarily translate into better performance however. As can be observed in Section 6.1 , the high CD value for a standard DFA means that it may take many lookups in memory to follow one transition.

Optimizing a DFA with single split-level drastically reduces SD and SR, as well as CD. Split-level reduces the dependency between states and therefore reduces SD. It also makes transitions more uniform, decreasing SR. With a much lower value for SD, $\mathrm{CD}$ is also much lower. For example, in an unoptimized DFA for the IMAP ruleset, SD $=0.619, \mathrm{CD}=0.041$, and $\mathrm{SR}=0.936$. However, for the DFA with single split-level, $\mathrm{SD}=0.111, \mathrm{CD}=0.003$, and $\mathrm{SR}=0.026$. The negative effects of the three metrics are almost eliminated with this split-level. The effects are even more significant when a second level of split-level is added. There is a slight drawback to this split-level however, in that multiple transitions may be needed to find a single next-state. In the case of single split-level, two transitions in a row may be needed, and in the case of multiple split-level, up to three transitions may be needed.

Table XII lists regular expression rule-sets and their properties. Due to state replication, regular expression DFA with similar numbers of patterns can have a drastically different number of states as seen in Table XII. We optimize the DFA in Table XII in one of two ways, either using the split-level optimization or the $D^{2} F A$ optimization. We inspect the values of the SD and SR metrics for the resulting DFA of both optimizations. We then use our character aware encoding to encode the DFA and display the resulting value requirements in Table XII.

Even after the split-level optimization, the values for state dependence (SD) and state irregularity (SR) are higher for these regular expression DFA than for the string based DFA presented earlier. This is due to the more complicated regular expression DFA structure. The metric values for the split-level optimization still show signs of state dependence and state irregularity. The $D^{2} F A$ optimization targets redundant transitions in any location of the DFA and therefore achieves a lower state dependence for the DFA in Table XII. 


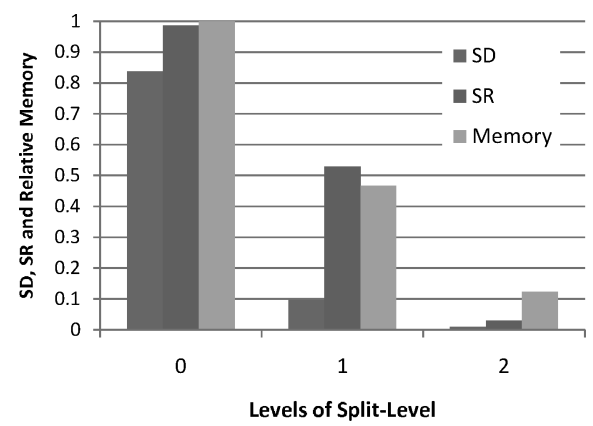

Fig. 23. Effects of split-level (memory based on SAM-FSM engine and relative to zero split-level).

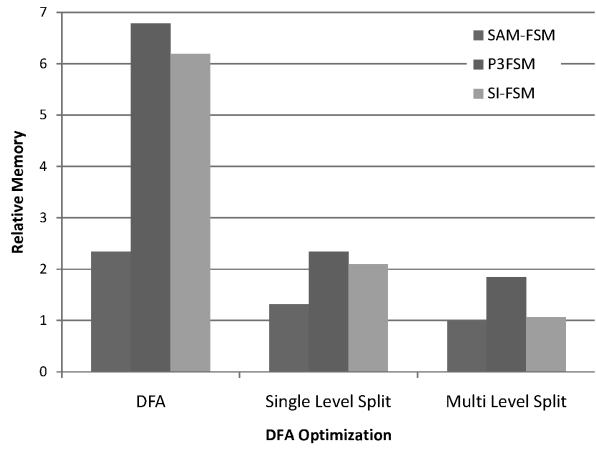

(a) Memory requirements for engines using different DFA optimizations

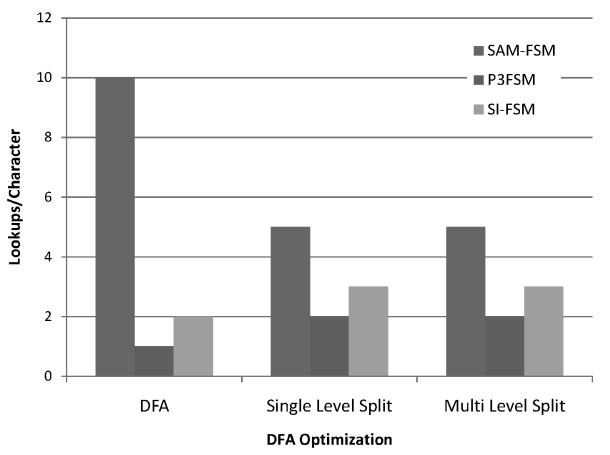

(b) Performance for engines using different DFA optimizations

Fig. 24. Memory and performance comparison of our engines.

Figure 23 shows SD, SR and memory for zero, one and two levels of split-level. The data comes from a 500 pattern DFA derived from Snort rulesets. The value of the memory is relative to no split-level. Without split-level, SD is around 0.84. After one level of split-level, SD decreases to 0.09 and when the DFA is completely normalized at two levels, SD reduces to less than 0.01 , which essentially means almost complete independence between states. This is because split-level reduces dependency between states as well as irregularity in transition distribution.

The most important observation to be made from Figure 23, is that the memory is reduced to about 47 percent of the original requirement after one level of split-level and to about 12 percent after two levels of split-level. These values for memory are especially exciting because the base value for memory with zero levels of split-level already takes state independence into account. So the decrease to 47 percent( 1 level) and then 12 percent( 2 levels) comes solely from the increase in state independence due to split-level.

\subsection{Pattern Matching Engines Evaluation}

Figure 24(a) shows the memory requirements for our three engines utilizing different DFAs. The DFAs implemented using the engines are a standard DFA, a DFA normalized using a single split-level and a DFA normalized using multiple split-level. The DFAs for Figure 24 as well as Figure 25 are derived from 500 random Snort patterns.

The memory requirement for SAM-FSM in Figure 24(a) is the lowest of all the engines. This is due to the self-addressable codes used to implement SAM-FSM. 


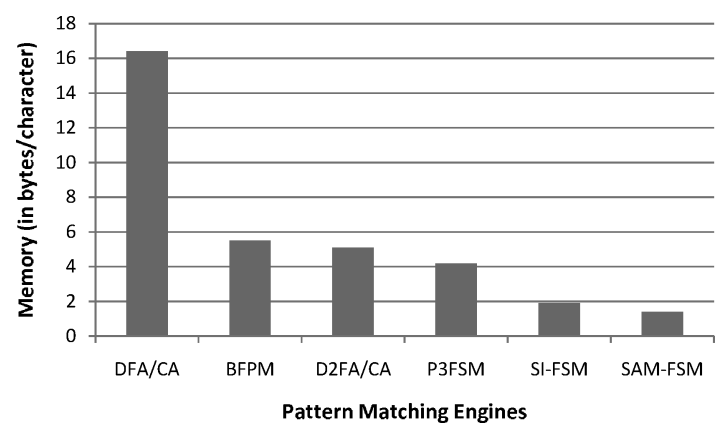

Fig. 25. Memory requirements for different engines.

Table XIII. Memory for Different DFAs Using a Character-
Aware Coding
\begin{tabular}{|l|c||c|}
\hline DFA Type & Ruleset & Memory(KB) \\
\hline \hline Traditional DFA & IMAP & 2.17 \\
& FTP & 4.16 \\
& Policy & 16.45 \\
& Web-CGI & 41.46 \\
\hline One Level split-level & IMAP & 0.75 \\
& FTP & 1.92 \\
& Policy & 5.77 \\
& Web-CGI & 13.96 \\
\hline Two Level split-level & IMAP & 0.34 \\
& FTP & 0.59 \\
& Policy & 1.90 \\
& Web-CGI & 3.97 \\
\hline
\end{tabular}

These codes take into account state-independence but are not implemented with the character-aware encoding optimization. This means that the performance of SAM-FSM will be worse than that of the other two engines, which are both character aware. The memory requirements for $P^{3} F S M$ are also slightly higher than those for SI-FSM. As expected, the memory requirements for all three engines decrease using the normalized DFA. This is because all three engines exploit state independence using selfaddressable encoding, and the split-level optimization increases state independence.

Figure 24(b) demonstrates the memory/performance tradeoff that is expected with any memory-based pattern matching engine. This figure shows the number of lookups required per character for each engine using all three DFA types. SAM-FSM requires the most lookups for all three DFAs because it does not utilize the character-aware encoding optimization. $P^{3} F S M$ requires the least lookups. This is because $P^{3} F S M$ is character aware. Notice that the number of lookups required for $P^{3} F S M$ is lower for the standard DFA than for the normalized DFA. This is because the un-normalized DFA are not split and therefore only one lookup per character is required with the DFA.

Table XIII shows the memory requirements to implement a character-aware memorybased engine for a standard DFA, single split-level DFA, and multiple split-level DFA. As expected, the standard DFA with no optimization applied requires the most memory of all rulesets. The split-level optimization reduces memory requirements substantially.

Table XIV shows the memory requirements for a character-aware encoding of different regex rule-sets using the split-level and $D^{2} F A$ optimizations. The memory requirements depend on the length of the code words for each state. The length in bits for each DFA is shown in the table. Because our encoding schemes depend on SD, any transition 
Table XIV. Memory Requirements for a Character Aware Encoding of Regex DFA

\begin{tabular}{|l|c|c|c|c|}
\hline & \multicolumn{2}{|c|}{ Split-Level } & \multicolumn{2}{c|}{ D2FA } \\
\hline Ruleset & Code Length (bits) & Mem(KB) & Code Length (bits) & Mem(KB) \\
\hline \hline POP3 & 18 & 0.21 & 16 & 0.19 \\
\hline SMTP & 23 & 0.23 & 19 & 0.19 \\
\hline Exploit & 199 & 58 & 162 & 49.76 \\
\hline VOIP & 127 & 40.25 & 109 & 32.77 \\
\hline
\end{tabular}

Table XV. Memory Requirements in Kilobytes for a Traditional DFA versus SI-FSM Using Different Sized Snort Rulesets

\begin{tabular}{|c|c|c|c|c|c|}
\hline \# Patterns & \# Characters & \# States & Traditional DFA (KB) & SI-FSM (KB) & Reduction (times) \\
\hline 5 & 98 & 93 & 21 & 0.43 & 48 \\
\hline 20 & 334 & 302 & 86 & 0.93 & 93 \\
\hline 50 & 663 & 568 & 181 & 1.57 & 115 \\
\hline 100 & 1291 & 1060 & 373 & 2.79 & 134 \\
\hline 200 & 2129 & 1601 & 563 & 4.17 & 135 \\
\hline 300 & 4313 & 3098 & 1189 & 8.11 & 147 \\
\hline 400 & 6722 & 4837 & 2012 & 12.86 & 156 \\
\hline 500 & 7637 & 5267 & 2191 & 14.05 & 156 \\
\hline 1000 & 14235 & 10477 & 4693 & 28.34 & 166 \\
\hline
\end{tabular}

reduction scheme, such as split-level and $D^{2} F A$, can be used to decrease the memory requirements for our encoding methods. This allows for an existing DFA-based regular expression-matching system to optimize their memory using our encoding methods. It is not necessary that the split-level optimization be specifically used, as in our engines. Our encoding optimizations simply exploit the independence between states to help compress the code word for each state and also allow for a speedy lookup of each next-state through our character-aware approach.

Table XV shows the memory requirements for a traditional DFA and our SI-FSM engine, using 5 to 1000 patterns. The traditional DFA is a simple memory-based DFA where 256 next state pointers are stored for each state. Table XV demonstrates two important attributes of our SI-FSM engine. First, Table XV shows that SI-FSM reduces memory requirements from the traditional DFA baseline by many times. Second, Table XV demonstrates that our SI-FSM engine memory scales predictably with the number of patterns, characters, and states. Even for a 1000-pattern ruleset, the number of times reduction from a traditional DFA for SI-FSM is not reduced.

Finally, all of the engines mentioned in this article are evaluated in terms of memory requirement in Figure 25. The engines evaluated are DFA/CA, which is a characteraware implementation of a standard DFA, BFPM [van Lunteren 2006], $D^{2} F A / C A$, which is a character aware implementation of a $D^{2} F A$ optimized DFA, $P^{3} F S M$, SIFSM, and SAM-FSM.

\subsection{Results Summary}

The results lead to three conclusions regarding our methodology. First, our characterization metrics are able to capture the correlation between DFA properties and memory/performance shown in Figure 22. This correlation is further verified by experimental evaluation in Figure 23. Second, state encoding techniques reveal the tradeoff between memory and performance in Figure 24, which indicates that a combination of different techniques must be used to achieve good memory and performance. Last, our engines improve memory efficiency compared to other engines reported from literature in Figure 25. These conclusions show that optimizing state encoding is a necessary compliment to traditional transition reduction techniques, and that our characterization can be used to guide pattern-matching engine design for desirable memory and performance. 


\section{RELATED WORK}

Pattern-matching for NIDS has been a deeply explored topic of research in recent years and many solutions have been presented. Some address exact string matching [Aho and Corasick 1975; Tan et al. 2006; Dixon et al. 2008] whereas others can be used to match regular expressions as well [Kumar et al. 2006; Becchi and Crowley 2007; Brodie et al. 2006; Smith et al. 2008a]. In this section, we focus on previous work related to three components of our methodology: DFA characterization, DFA optimization, and memory-based engines.

A substantial amount of work [Tseng et al. 2005; Dharmapurikar et al. 2004; Sourdis et al. 2006; Aldwairi et al. 2005; Cho et al. 2002; Yu et al. 2004] has been performed in the area of pattern-matching. Most of the existing work is not suitable for new generations of NIDSs that require simultaneous matching of thousands of patterns. DFA-based pattern matching has a huge memory requirement, however few works analyze DFA characteristics that cause severe memory space problems. Our preliminary work [Vespa and Weng 2009a] presents two metrics to characterize state independency and irregularity, however it does not address input symbol distribution and how to apply to regular expression DFA. Based on this preliminary work, this article presents a general methodology, which consists of theoretical DFA characterization, encoding optimization, and implementation architecture for both string and regular expression DFA. Dixon et al. [2008] present a theoretic automata analysis of a special bit-split language. However, our approach applies to the general DFA problem. Becchi et al. [2008] present a workload to evaluate different regular expression-matching engines. The workload models regular expression patterns by formally characterizing the level of expressiveness within rulesets. The proposed workload model [Becchi et al. 2008] provides a missing benchmark to fairly compare different pattern-matching algorithms. However, our characterization metrics aim to understand the relationship between DFA characteristics and memory requirements, this understanding will help us to find better encoding techniques.

The D2FA scheme [Kumar et al. 2006] performs transition reduction to minimize the number of transitions for each state. Many common transitions between states are removed and replaced with single default transitions. This removes redundancy, leaving the DFA with only unique transitions.

Becchi and Crowley [2007] introduce an optimization for which the compression is not limited by the bounds of sequential default transitions of Kumar et al. [2006]. It achieves worst-case processing time of $2 \mathrm{~N}$ transitions yet still attains memory reduction similar to that of Kumar et al. [2006]. Becchi and Crowley [2007] also present a method for alphabet reduction, which allows for better memory compression.

van Lunteren [2006] introduced a memory-based string-matching engine, BFPM, which uses a prioritized list of state transition rules stored in memory. These state transition rules are based on the B-FSM implementation of finite state machine, which uses an "optimized version of the Balanced Routing Table(BART) search algorithm." BFPM focusses on encoding optimization and memory-based engine architecture. The prioritized rules are similar to our split-level technique, for two reasons. First, many backward transitions are replaced with a single low priority rule, as in our single split-level. Second, the DFA is clustered into sections in order to simplify rule lookup within each section, as in our multiple split-level. Occasionally, lookup needs to take place between the clustered sections, as is done between our primary and secondary memories. A memory-based engine such as $P^{3} F S M$ however, only has up to two-level prioritization for split-level DFA so we do not require parallel lookup for any transitions.

Bit-Split [Tan et al. 2006] utilizes encoding optimization to implement a memorybased string matching engine. It optimizes the DFA input to the engine by splitting 
the DFA into multiple machines, each of which processes only a single bit of the input stream. The key differences between Bit-Split engine and our work are that our memory-based architecture is designed to be more general and can be implemented in most memory-based systems. Our engines also utilize a single DFA rather than multiple DFA running in parallel to handle each bit of the input. This is partly responsible for our ability to implement our engines independently of a specific platform.

The concept of storing many or all next-state pointers for each state in a single word as we do in our state codes, has been explored by work such as Hayes and Luo [2007]. In this work, each transition is labeled with an input character. If no valid labeled transition exists for the current input, a default transition is followed. Unlike our work however, Hayes and Luo [2007] requires n (fan-out of current state) labeled transitions to be searched in parallel, leveraging expensive hardware (CAM). In our work, based on the current input, our engine needs examine only one labeled transition to determine the next state. Our character-aware encoding stores labeled transitions for each character in the same entry of all state codes. This allows us to achieve this exceptionally simple processing capability, making our engines fast even in simple software.

\section{CONCLUSION}

In this work, we have introduced a methodology that consists of theoretical DFA characterization, encoding optimization, and implementation architecture for scalable pattern-matching in network intrusion detection systems. For DFA characterization, we have introduced three metrics: state independence, character distribution, and state irregularity. The state independence and state irregularity metrics map out DFA characteristics that affect memory, while character distribution demonstrates the same for performance. With this theoretical understanding of DFA, we present three encoding optimizations that specifically target the characteristics exposed by our metrics. Selfaddressable encoding exploits state independence to minimize memory and characteraware encoding resolves the problem of DFA lookup. Split-level is implemented to minimize state irregularity, which also enhances the effects of self-addressable encoding. To demonstrate the effectiveness of optimization techniques, we have presented three memory-based engines: Self Addressable Memory-Based Finite State Machine, Portable Predictive Pattern Matching Finite State Machine, Simple Instruction Finite State Machine. These engines all require minimal resources, are easy to implement, and are platform-independent.

We believe that our methodology bridges many gaps between existing heuristic minimization methods, in order to help solve the overall problem of memory-based, deterministic pattern matching. We also believe our work is a vitally important step in a collaborative effort to develop the high-performance, adaptable, and memory-efficient pattern matching that is needed by modern-day network intrusion detection systems.

\section{REFERENCES}

Aнo, A. And Corasick, M. 1975. Efficient string matching: An aid to bibliographic search. Comm. ACM 18, 333-340.

Aldwairi, M., Conte, T., and Franzon, P. 2005. Configurable string matching hardware for speeding up intrusion detection. SIGARCH Comput. Archit. News 33, 1, 99-107.

BECCHI, M. AND CROwLEY, P. 2007. An improved algorithm to accelerate regular expression evaluation. In Proceedings of the ACM/IEEE Symposium on Architectures for Networking and Communications Systems (ANCS). 145-154.

Becchi, M., Franklin, M., and Crowley, P. 2008. A workload for evaluating deep packet inspection architectures. In Proceedings of the IEEE International Symposium on Workload Characterization. 79-89.

Brodie, B. C., Taylor, D. E., ANd Cytron, R. K. 2006. A scalable architecture for high-throughput regularexpression pattern matching. SIGARCH Comput. Archit. News 34, 2, 191-202. 
Bu, L. AND Chandy, J. A. 2004. FPGA based network intrusion detection using content addressable memories. In Proceedings of the 12th Annual IEEE Symposium on Field-Programmable Custom Computing Machines (FCCM'04). 316-317.

Cho, Y. H., Navab, S., AND Mangione-Smith, W. H. 2002. Specialized hardware for deep network packet filtering. In Proceedings of the 12th International Conference on Field-Programmable Logic and Applications (FPL). Springer-Verlag, 452-461.

Denning, D. 1987. An intrusion-detection model. IEEE Trans. Softw. Eng. 13, 2, 222-232.

Dharmapurikar, S., Krishnamurthy, P., Sproull, T. S., ANd Lockwood, J. W. 2004. Deep packet inspection using parallel Bloom filters. IEEE Micro 24, 1, 52-61.

DiXon, R., EĞECioĞLU, O., AND SHERwOOD, T. 2008. Automata-theoretic analysis of bit-split languages for packet scanning. In Proceedings of the 13th International Conference on Implementation and Applications of Automata (CIAA). Springer-Verlag, 141-150.

HAYES, C. L. AND LUO, Y. 2007. DPICO: a high speed deep packet inspection engine using compact finite automata. In Proceedings of the 3rd ACM/IEEE Symposium on Architecture for Networking and Communications Systems (ANCS). 195-203.

Herisson, J., PaYen, G., ANd Gherbi, R. 2007. A 3D pattern matching algorithm for DNA sequences. Bioinformatics, 680-686.

Kumar, S., Dharmapurikar, S., Yu, F., Crowley, P., and Turner, J. 2006. Algorithms to accelerate multiple regular expressions matching for deep packet inspection. In Proceedings of ACM SIGCOMM. 339-350.

LU, Y., LU, S., AND RAM, J. L. 2006. Fast search in DNA sequence databases using punctuation and indexing. In Proceedings of the 2nd IASTED International Conference on Advances in Computer Science and Technology (ACST). ACTA Press, 351-356.

Roesch, M. 1999. Snort - lightweight intrusion detection for networks. In Proceedings of the 13th Systems Administration Conference.

Smith, R., Estan, C., AND JHA, S. 2008a. XFA: Faster signature matching with extended automata. In Proceedings of the IEEE Symposium on Security and Privacy. $187-201$.

Smith, R., Estan, C., Jha, S., And Kong, S. 2008b. Deflating the big bang: fast and scalable deep packet inspection with extended finite automata. In Proceedings of the ACM SIGCOMM Conference on Data Communication (SIGCOMM). 207-218.

SNORT. 2009. Snort Rule Database. Snort. http://www.snort.org/pub-bin/downloads.cgi.

Soewito, B., Vespa, L., Mahajan, A., Weng, N., AND Wang, H. 2009. Self addressable memory-based FSM (SAMFSM): A scalable intrusion detection engine. IEEE Network (Special Issue on Recent Developments in Network Intrusion Detection) 23, 1, 14-21.

Soewito, B. And Weng, N. 2007. Methodology for evaluating DNA pattern searching algorithms on multiprocessors. In Proceedings of the 7th IEEE International Conference on Bioinformatics and Bioengineering, 570-577.

Sourdis, I., Dimopoulos, V., Pnevmatikatos, D., and Vassiliadis, S. 2006. Packet pre-filtering for network intrusion detection. In Proceedings of the ACM/IEEE Symposium on Architecture for Networking and Communications Systems (ANCS). 183-192.

TAN, L., BRotherton, B., AND Sherwood, T. 2006. Bit-split string-matching engines for intrusion detection and prevention. ACM Trans. Archit. Code Optim. 3, 1, 3-34.

TsenG, K.-K., LiN, Y.-D., LEE, T.-H., AND LAI, Y.-C. 2005. A parallel automaton string matching with pre-hashing and root-indexing techniques for content filtering coprocessor. In Proceedings of the IEEE International Conference on Application-Specific Systems, Architecture Processors (ASAP). 113-118.

Tuck, N., Sherwood, T., Calder, B., And Varghese, G. 2004. Deterministic memory-efficient string matching algorithms for intrusion detection. In Proceedings of the IEEE International Conference on Computer Communications (INFOCOM). 333-340.

van Lunteren, J. 2006. High-performance pattern-matching for intrusion detection. In Proceedings of the IEEE International Conference on Computer Communications (INFOCOM), 1-13.

VARghese, G. 2005. Network Algorithmics: An Interdisciplinary Approach to Designing Fast Networked Devices 1st Ed. Morgan Kaufmann.

Vespa, L., Mathew, M., AND Weng, N. 2009. P3FSM: Portable predictive pattern matching finite state machine. In Proceedings of the 20th IEEE International Conference on Application-specific Systems, Architectures and Processors. 219-222.

Vespa, L. AND Weng, N. 2009a. Deterministic finite automata characterization for memory-based pattern matching. In Proceedings of the International Conference on Information, Communications, and Signal Processing (ICICS). 268-282. 
VESPA, L. AND WENG, N. 2009b. Split-DFA (SDFA) for scalable pattern matching in network security. In Proceedings of the International Conference on Security and Management (SAM). 469-474.

Yu, F., Chen, Z., Diao, Y., Lakshman, T., and Katz, R. H. 2006. Fast and memory-efficient regular expression matching for deep packet inspection. In Proceedings of the ACM/IEEE Symposium on Architecture for Networking and Communication Systems (ANCS). 93-102 .

YU, F., KatZ, R. H., AND LAKSHMAN, T. V. 2004. Gigabit rate packet pattern-matching using tcam. In Proceedings of the 12th IEEE International Conference on Network Protocols (ICNP), 174-183.

Received October 2010; revised March 2010, August; accepted October 2010 